\title{
Reduction of general two-loop self-energies to standard scalar integrals内
}

\author{
G. Weiglein?], R. Scharf, M. Böhm \\ Physikalisches Institut der Universität Würzburg, \\ Am Hubland, D-97074 Würzburg, Federal Republic of Germany
}

February 1993

\begin{abstract}
A method is presented for reducing general two-loop self-energies to standard scalar integrals in massive gauge theories with special emphasis on the electroweak Standard Model (SM). We develop a technique for treating the tensor structure of two-loop integrals appearing in selfenergy calculations. It is used together with the symmetry properties of the integrals to obtain a result in terms of a small number of standard scalar integrals. The results are valid for arbitrary values of the invariant momentum $p^{2}$, all particle masses, the space-time dimension $D$ and the gauge parameters $\xi_{i}(i=\gamma, Z, W)$. The algebraic structure of the results clearly displays the gauge dependence of the considered quantities and allows to perform very stringent checks. We explicitly verify Slavnov-Taylor identities by calculating several thousand Feynmandiagrams and adding them up algebraically. As an application of our algorithm we calculate the light fermion contributions to the two-loop gauge boson self-energies of the electroweak SM. We study their gauge dependence and discuss the occurring standard integrals.
\end{abstract}

\footnotetext{
1 Accepted for publication in Nuclear Physics B

${ }^{2}$ E-mail address: weiglein@vax.rz.uni-wuerzburg.d400.de
} 


\section{Introduction}

The $e^{+} e^{-}$colliders LEP100 and SLC started a new era of precision measurements which allow to test the electroweak Standard Model (SM) on its quantum level. In order to match the experimental precision radiative corrections have to be incorporated into the theoretical predictions. During the last years many calculations of electroweak virtual corrections have been carried out at the one-loop level. For the high precision experiments at LEP100, however, first order corrections alone are inadequate. Leading second order effects are often taken into account by means of renormalization group methods, but rather limited results have been obtained for irreducible virtual two-loop corrections.

In the study of these contributions the self-energies play a central role. These so-called oblique corrections are universal, i.e. process-independent, in contrast to the processspecific contributions due to vertices, boxes and bremsstrahlung. A number of authors [1, 2] recently stressed the importance of the oblique corrections for the analysis of precision experiments and in reformulations of electroweak radiative corrections using effective lagrangians. In view of the measurements at LEP100, in particular a precise calculation of the Z-boson self-energy is of interest.

In the SM so far no complete calculation of a two-loop self-energy has been carried out. This fact is due to the complicated structure and large number of the Feynman diagrams contributing at the two-loop level. Results were obtained treating the limiting cases of a heavy fermion doublet [3], a heavy top quark [4] and a large Higgs mass [5]. The result given in [3] was used in [6] for studying the resummation of effects due to fermion doublets with large mass splitting.

In view of the fact that the mass expected for the top-quark is of the order of the heavy gauge boson masses and that almost no restrictions can be imposed on the Higgs mass a calculation allowing for general values of the top and Higgs mass, the gauge boson masses and the invariant momentum $p^{2}$ might be of interest.

In this paper we present a systematic way for treating all two-loop self-energies. It is applicable for gauge bosons, scalars, gauge boson-scalar mixing and fermions. In our discussion we focus on the electroweak SM, but the method is valid for any renormalizable model. The strategy we have adopted is to reduce the amplitudes algebraically as far as possible. We develop a technique for the tensor decomposition of two-loop self-energy integrals. This generalizes the results for one-loop integrals worked out by Passarino and Veltman [7]. Using this technique and the symmetry properties of the integrals we obtain a result in which the Feynman amplitudes are given in terms of standard scalar two-loop integrals. This is in analogy to one-loop calculations where the Feynman amplitudes are expressed in terms of the standard integrals $A_{0}, B_{0}, C_{0}$ and $D_{0}$ defined in [8]. We will show that the reduction to the specified class of integrals is possible for every two-loop self-energy.

The calculations are performed for arbitrary values of all particle masses, the invariant momentum $p^{2}$ and the space-time dimension $D$. We work in a general $R_{\xi}$-gauge specified by one gauge parameter $\xi_{i}(i=\gamma, Z, W)$ for each vector boson. This allows to study the gauge dependence of the calculated quantities. The gauge dependence of basic electroweak corrections has recently found considerable interest and was studied at one-loop order by a number of authors [2, 9, 10, 11]. At the two-loop level this issue is of even greater 
importance since as long as the calculation of a complete process, which necessarily is gauge invariant, is out of reach it is crucial to know the gauge dependence of the results. We will show that our method of representing the results in terms of two-loop standard integrals very clearly displays the gauge dependence of the considered quantities. It can be read off directly from the algebraic result. There is no need for using an explicit analytical or numerical expression of the standard integrals.

We show that our algorithm for treating the two-loop self-energies is well suited for calculations involving a large number of Feynman diagrams. The results consist of a relatively small number of standard integrals which can conveniently be studied for further evaluation. In contrast to that a direct evaluation of the tensor integrals would in general involve a large number of integrals, each one to be treated separately. The results we obtain are transparent and have the benefit that very stringent checks can easily be performed on them. In analogy to the investigation of the gauge dependence we can check Slavnov-Taylor identities directly at the algebraic level. This test is exact, i.e. free of any numerical uncertainty. We implemented our algorithm in the computer-algebra program Two Calc [12]. In order to check its reliability we explicitly verify Slavnov-Taylor identities for the self-energies of the $\gamma Z$-system involving several thousand Feynman diagrams. Every graph is calculated separately and the results are summed up algebraically.

As an application we treat the light fermion contributions to the two-loop gauge boson self-energies in the SM. Considering the results obtained in one-loop order the light fermions are expected to yield a significant contribution to the complete result. We give the results for the $\gamma, Z$ and $W$ self-energies in terms of two-loop standard integrals for general values of $p^{2}$, the gauge boson masses and the Higgs mass. We study the gauge dependence of these amplitudes and classify them in several subsets according to their behavior under gauge transformations. All standard integrals appearing in the results of the light fermion contributions can be solved analytically leading to an expression in terms of polylogarithmic functions. We worked this out explicitly. The results will be presented in a related paper [13].

The paper is organized as follows: In sect. 2 we classify the two-loop self-energies according to their topologies and define the relevant quantities. Sect. 3 is concerned with the symmetry properties of the two-loop integrals. In sect. 4 we develop a technique for the tensor decomposition of two-loop integrals and show that it is applicable for every integral which arises in the calculation of two-loop self-energies. In sect. 5 we consider relations used for minimizing the number of occurring standard integrals. Sects. 6 and 7 deal with the computer-algebraic realization of the algorithm and its use for verifying Slavnov-Taylor identities in the electroweak SM. In sect. 8 we give results for the two-loop photon self-energy in QED and for the light fermion contributions to the two-loop gauge boson self-energies in the electroweak SM. The properties of these results are discussed. In the appendix we give a result for the $W$ self-energy and list several reduction formulae needed for the calculations performed in this paper. 


\section{Classification of two-loop self-energies}

The two-loop self-energies can be classified according to the topologies of the corresponding Feynman diagrams. All self-energy topologies which can occur in renormalizable gauge theories are shown in fig. 2.1. We have listed the one-particle irreducible topologies, where as usual diagrams containing tadpole lines are included. Furthermore nine reducible topologies exist which correspond to products of one-loop self-energies. In the following we will call the first eight topologies in fig. 2.1 "generic" two-loop topologies.

We focus on the unrenormalized self-energies, a proper renormalization can be done after the algebraic calculation has been carried out. In order to make the integrals mathematically meaningful we use dimensional regularization and work in an arbitrary spacetime dimension $D$.

Inserting fields into the topologies and applying the Feynman rules for propagators and vertices leads to the Feynman amplitudes. It is convenient to deal with scalar quantities, i.e. to begin with a tensor decomposition. For the gauge boson self-energies it reads

$$
\Sigma_{\mu \nu}^{\alpha, \beta}(p)=\left(-g_{\mu \nu}+\frac{p_{\mu} p_{\nu}}{p^{2}}\right) \Sigma_{T}^{\alpha, \beta}\left(p^{2}\right)-\frac{p_{\mu} p_{\nu}}{p^{2}} \Sigma_{L}^{\alpha, \beta}\left(p^{2}\right),
$$

from which the transverse part $\Sigma_{T}^{\alpha, \beta}\left(p^{2}\right)$ and the longitudinal part $\Sigma_{L}^{\alpha, \beta}\left(p^{2}\right)$ can easily be extracted:

$$
\Sigma_{T}^{\alpha, \beta}\left(p^{2}\right)=\frac{1}{D-1}\left(-g^{\mu \nu}+\frac{p^{\mu} p^{\nu}}{p^{2}}\right) \Sigma_{\mu \nu}^{\alpha, \beta}(p) ; \quad \Sigma_{L}^{\alpha, \beta}\left(p^{2}\right)=-\frac{p^{\mu} p^{\nu}}{p^{2}} \Sigma_{\mu \nu}^{\alpha, \beta}(p),
$$

$p$ is the external momentum, $D$ the space-time dimension, $\alpha, \beta=\gamma, Z$ for the $(\gamma Z)$-system and $\alpha=W, \beta=W$ for the $W$-boson.

We write for the mixing of gauge bosons and unphysical Higgs-fields

$$
\Sigma_{\mu}^{\alpha, i}(p)=p_{\mu} \Sigma^{\alpha, i}\left(p^{2}\right) .
$$

Consequently we have

$$
\Sigma^{\alpha, i}\left(p^{2}\right)=\frac{p^{\mu}}{p^{2}} \Sigma_{\mu}^{\alpha, i}(p)
$$

where $i=\chi, \varphi$ for the $(\gamma Z)$-system and the $W$-boson, respectively.

The fermion self-energies can be decomposed into a vector, an axial vector, a scalar and a pseudoscalar part according to

$$
\Sigma(p)=\not p \Sigma_{V}\left(p^{2}\right)+\not p \gamma_{5} \Sigma_{A}\left(p^{2}\right)+m \Sigma_{S}\left(p^{2}\right)+m \gamma_{5} \Sigma_{P}\left(p^{2}\right),
$$

where $m$ is the mass of the fermion and mixing effects have been suppressed for simplicity of notation. We obtain

$$
\Sigma_{V}\left(p^{2}\right)=\frac{1}{4 p^{2}} \operatorname{Tr}(\not p \Sigma(p)) ; \quad \Sigma_{A}\left(p^{2}\right)=\frac{1}{4 p^{2}} \operatorname{Tr}\left(\gamma_{5} \not p \Sigma(p)\right)
$$

and

$$
\Sigma_{S}\left(p^{2}\right)=\frac{1}{4 m} \operatorname{Tr}(\Sigma(p)) ; \quad \Sigma_{P}\left(p^{2}\right)=\frac{1}{4 m} \operatorname{Tr}\left(\gamma_{5} \Sigma(p)\right) .
$$


Figure 2.1: The 20 topologies possible for two-loop self-energies in renormalizable gauge theories. 
Adding the inverse of the zeroth order propagator to the self-energies of all orders (including the tadpole amplitudes) leads to the truncated one-particle irreducible twopoint functions. For the Higgs field being a Lorentz scalar this yields

$$
\Gamma^{H}(p)=i\left(p^{2}-m_{H}^{2}\right)+i \Sigma^{H}\left(p^{2}\right)
$$

and for the Lorentz tensors we use the decompositions specified above. The corresponding propagators are obtained as the inverse of these two-point functions. For the neutral gauge bosons we have to consider matrices. The transverse part of the inverse propagator matrix reads

$$
D_{T}^{-1}=i\left(\begin{array}{ll}
p^{2}+\Sigma_{T}^{\gamma \gamma}\left(p^{2}\right) & \Sigma_{T}^{\gamma Z}\left(p^{2}\right) \\
\Sigma_{T}^{\gamma Z}\left(p^{2}\right) & p^{2}-M_{Z}^{2}+\Sigma_{T}^{Z Z}\left(p^{2}\right)
\end{array}\right)
$$

from which the propagators

$$
D_{T}=\left(\begin{array}{cc}
\Delta_{T}^{\gamma \gamma} & \Delta_{T}^{\gamma Z} \\
\Delta_{T}^{\gamma Z} & \Delta_{T}^{Z Z}
\end{array}\right)
$$

follow by matrix inversion.

We recall that in the $R_{\xi}$-gauges the lowest order gauge boson propagators can be written as

$$
D_{\mu \nu}^{i}(k)=\frac{-i g_{\mu \nu}}{\left[k^{2}-m_{i}^{2}\right]}+\frac{i\left(1-1 / \xi_{i}\right) k_{\mu} k_{\nu}}{\left[k^{2}-m_{i}^{2} / \xi_{i}\right]\left[k^{2}-m_{i}^{2}\right]},
$$

where $i=\gamma, Z, W$. The parameters $\xi_{i}$ associated with these fields specify the gauge. They can be chosen independently. The 't Hooft-Feynman gauge is realized by setting $\xi_{i}=1$, $\xi_{i} \rightarrow \infty$ defines the Landau gauge while the limit $\xi_{i} \rightarrow 0$ corresponds to the unitary gauge. The unphysical scalar and ghost propagators are of the form $i\left[k^{2}-m_{i}^{2} / \xi_{i}\right]^{-1}$. The fermion propagators can be written as $i(\not k+m)\left[k^{2}-m^{2}\right]^{-1}$.

In the following we will use the shorthand notation

$$
\langle\langle\ldots\rangle\rangle=\int \frac{d^{D} q_{1}}{i \pi^{2}(2 \pi \mu)^{D-4}} \int \frac{d^{D} q_{2}}{i \pi^{2}(2 \pi \mu)^{D-4}}(\ldots),
$$

where $q_{1}$ and $q_{2}$ are the integration momenta of the loop integrals and $\mu$ is an arbitrary reference mass.

The Feynman amplitudes we are concerned with can therefore be written as

$$
\left\langle\left\langle\frac{\cdots}{\left[k_{1}^{2}-m_{1}^{2}\right]\left[k_{2}^{2}-m_{2}^{2}\right] \cdots\left[k_{\ell}^{2}-m_{\ell}^{2}\right]}\right\rangle\right\rangle,
$$

where $k_{j}$ is the momentum of the $j$-th propagator and $m_{j}$ its mass, $j=1, \ldots, \ell$. The $m^{2} / \xi_{i}$-terms occurring in the propagators given above are simply treated as mass parameters. In (2.13) it is understood that the masses carry a small negative imaginary part.

The numerator of the Feynman amplitude in general has a complicated structure being a function of the two integration momenta, the external momentum $p$, the particle 
Figure 2.2: The topology of the "master integral"

masses and the gauge parameters $\xi_{i}$. It will be a central issue of this paper to show how all Feynman amplitudes can be reduced to a form where the numerator consists only of quantities which are independent of the integration momenta and can therefore be pulled out of the integral. The denominator is still of the form (2.13). This means that the amplitudes can be expressed in terms of a class of scalar two-loop integrals

$$
T_{i_{1} i_{2} \ldots i_{\ell}}\left(p^{2} ; m_{1}^{2}, m_{2}^{2}, \ldots, m_{\ell}^{2}\right)=\left\langle\left\langle\frac{1}{\left[k_{i_{1}}^{2}-m_{1}^{2}\right]\left[k_{i_{2}}^{2}-m_{2}^{2}\right] \cdots\left[k_{i_{\ell}}^{2}-m_{\ell}^{2}\right]}\right\rangle\right\rangle,
$$

which we call $T$-integrals. This is reminiscent of the well known result that every one-loop amplitude can be reduced to the basic scalar integrals $A_{0}, B_{0}, C_{0}$ and $D_{0}$ [7, 8]. It should be noted, however, that it is by far not obvious that such a reduction is possible in the general two-loop case and we only claim it for self-energies.

We used the double index notation $T_{i_{1} i_{2} \ldots i_{\ell}}$ to indicate that the subindices of the $T$ integrals refer to the corresponding momenta $k_{i_{1}}, k_{i_{2}}, \ldots k_{i_{\ell}}$. The masses are only explicitly written as arguments if confusion is possible. If a propagator has mass zero, we indicate this with a prime at the corresponding subindex and drop the zero in the list of arguments, e.g.

$$
T_{1^{\prime} 234}\left(p^{2} ; m_{a}^{2}, m_{b}^{2}, m_{c}^{2}\right)=\left\langle\left\langle\frac{1}{k_{1}^{2}\left[k_{2}^{2}-m_{a}^{2}\right]\left[k_{3}^{2}-m_{b}^{2}\right]\left[k_{4}^{2}-m_{c}^{2}\right]}\right\rangle\right\rangle .
$$

The dependence on $p^{2}$ will be suppressed in the following.

The topologies listed in fig. 2.1 can also be used to represent the $T$-integrals. A line in the topology carrying zero momentum contributes only a factor $\left(-1 / m_{i}^{2}\right)$ which is irrelevant for the scalar integral. The topologies 6 and 7 in fig. 2.1 therefore represent the same type of $T$-integral. The first two topologies in fig. 2.1 are the most general ones since all scalar integrals corresponding to the other topologies can be obtained from these by omitting factors $\left[k_{j}^{2}-m_{j}^{2}\right]$ in the denominator or equivalently shrinking the corresponding lines in the topology to a point. The integral corresponding to the first topology, being the only one where five propagators with different momenta occur, is sometimes called the "master integral"

$$
T_{12345}=\left\langle\left\langle\frac{1}{\left[k_{1}^{2}-m_{1}^{2}\right]\left[k_{2}^{2}-m_{2}^{2}\right]\left[k_{3}^{2}-m_{3}^{2}\right]\left[k_{4}^{2}-m_{4}^{2}\right]\left[k_{5}^{2}-m_{5}^{2}\right]}\right\rangle\right\rangle,
$$

where the momenta are labeled as indicated in fig. 2.2. In the following we do not manifestly impose momentum conservation, but keep the overcomplete set of momenta $k_{1}, \ldots, k_{5}$. This will be convenient especially for treating the symmetry properties of 
the integrals and performing the tensor reductions. It is easy to reexpress the momenta $k_{1}, \ldots, k_{5}$ by the external momentum $p$ and the integration momenta $q_{1}$ and $q_{2}$ :

$$
k_{1}=q_{1}, k_{2}=q_{1}+p, k_{3}=q_{2}-q_{1}, k_{4}=q_{2}, k_{5}=q_{2}+p .
$$

With this convention, the integral corresponding to the second topology in fig. 2.1, for example, can be written as $T_{11234}$. We will see below that equivalent representations can be obtained by certain permutations of the indices.

\section{Symmetries of the two-loop integrals}

As stated above we start with a tensor decomposition (eqs. (2.1), (2.3) and (2.5), respectively) in order to obtain scalar quantities. The contraction of Lorentz indices, reduction of the Dirac algebra and evaluation of Dirac traces can be worked out like in the one-loop case. This results in scalar products of momenta $\left(k_{i} \cdot k_{j}\right),\left(k_{i} \cdot p\right), p^{2}$ in the numerator of the Feynman amplitude. The denominator is unchanged. We now implicitly use momentum conservation and express all scalar products as sums of momentum squares, e.g.

$$
\left(k_{1} \cdot p\right)=\frac{1}{2}\left(k_{2}^{2}-k_{1}^{2}-p^{2}\right) .
$$

Subsequently all $k_{i}^{2}$ appearing both in the numerator and the denominator are canceled via

$$
k_{i}^{2}=\left(k_{i}^{2}-m_{i}^{2}\right)+m_{i}^{2} .
$$

The application of this procedure directly leads to $T$-integrals, if all $k_{i}^{2}$ appearing in the numerator can be canceled. If not, we obtain another type of integral which contains squares of momenta in the numerator not occurring in the denominator:

$$
Y_{j k l \ldots}^{i \ldots}=\left\langle\left\langle k_{i}^{2} \cdots \Delta_{j k l \ldots}\right\rangle\right\rangle, \quad i, j, k, l=1, \ldots, 5, \quad i \neq j, k, l .
$$

Here we used the shorthand notation

$$
\Delta_{j k l \ldots}=\frac{1}{\left[k_{j}^{2}-m_{j}^{2}\right]\left[k_{k}^{2}-m_{k}^{2}\right]\left[k_{l}^{2}-m_{l}^{2}\right] \cdots} .
$$

We now have obtained a representation of the amplitudes in terms of scalar $T$ - and $Y$-integrals. These integrals, however, are not independent of each other. The number of occurring integrals can considerably be reduced by taking into account their symmetries with respect to permutation of the $k_{i}^{2}$ or, equivalently, of the corresponding indices.

All $T$ - and $Y$-integrals are invariant under the permutations

$$
\text { (12)(45), (14)(25), (15)(24). }
$$

Here, as always, $k_{1}, \ldots, k_{5}$ are labeled according to (2.17). Application of the first permutation yields for example

$$
\begin{aligned}
T_{1234}\left(p^{2} ; m_{a}^{2}, m_{b}^{2}, m_{c}^{2}, m_{d}^{2}\right) & =T_{1235}\left(p^{2} ; m_{b}^{2}, m_{a}^{2}, m_{c}^{2}, m_{d}^{2}\right) \\
& =\left\langle\left\langle\frac{1}{\left[k_{1}^{2}-m_{b}^{2}\right]\left[k_{2}^{2}-m_{a}^{2}\right]\left[k_{3}^{2}-m_{c}^{2}\right]\left[k_{5}^{2}-m_{d}^{2}\right]}\right\rangle\right\rangle .
\end{aligned}
$$


The validity of the symmetries listed in (3.5) can most easily be seen for the "master integral" (fig. 2.2) or can be checked using (2.17) and the invariance properties of the integrals with respect to changes of the integration momenta.

Additional symmetry relations hold if an index does not occur in the integral, e.g.

(23) if 1 is absent.

The symmetry relations (3.5) and (3.7) are used to map every integral onto a standard representative, i.e. all integrals which are related by symmetry transformations are brought to the same form.

\section{Tensor reduction for two-loop integrals}

As claimed above, all self-energy amplitudes can be expressed by an independent set of $T$-integrals. This means that all $Y$-integrals can further be reduced. To achieve this, we rewrite the $Y$-integrals in terms of tensor integrals and perform a tensor decomposition.

As a simple example we consider the integral

$$
Y_{2345}^{1}=\left\langle\left\langle k_{1}^{2} \Delta_{2345}\right\rangle\right\rangle .
$$

Insertion of

$$
k_{1}^{2}=\left(k_{2}^{2}-m_{2}^{2}\right)+\left(m_{2}^{2}+p^{2}\right)-2\left(p \cdot k_{2}\right)
$$

yields

$$
Y_{2345}^{1}=T_{345}+\left(m_{2}^{2}+p^{2}\right) T_{2345}-2 p_{\mu}\left\langle\left\langle k_{2}^{\mu} \Delta_{2345}\right\rangle\right\rangle .
$$

Now one has to perform a tensor decomposition for the integral

$$
S_{2345}^{2, \mu}=\left\langle\left\langle k_{2}^{\mu} \Delta_{2345}\right\rangle\right\rangle .
$$

It is obvious that the Passarino-Veltman procedure [7] used for one-loop integrals, i.e. the ansatz

$$
S_{2345}^{2, \mu}=p^{\mu} S\left(p^{2}\right),
$$

does not lead to simpler integrals in this case. To determine the scalar quantity $S\left(p^{2}\right)$ one has to contract with $p_{\mu}$, but it is not possible to cancel the resulting scalar product $\left(p \cdot k_{2}\right)$, since it is not expressible as a sum of squared momenta occurring in the denominator of the integral. This is due to the fact that the momenta $p$ and $k_{2}$ belong to a four-vertex in the topology $\Delta_{2345}$ (see fig. 4.1). This is a typical feature of two-loop topologies, whereas in one-loop order only three-vertices occur in the loop integral and the scalar products can always be canceled.

In our approach we not only work with the tensor structure of the integral with respect to the external momentum $p$ but also perform decompositions with respect to a subloop. In general one can write for a subloop $s^{\mu}$ which has the structure of a first-rank tensor

$$
s^{\mu}=p_{1}^{\mu} s_{1}+p_{2}^{\mu} s_{2}+\ldots
$$


where $s_{1}, s_{2}, \ldots$ are Lorentz-scalars and $p_{1}, p_{2}, \ldots$ are the independent external momenta of the subloop.

At first sight it is not obvious that the scalar products resulting from this decomposition can be canceled. Moreover, as is well known from one-loop calculations, contracting (4.6) with $p_{1 \mu}, p_{2 \mu}, \ldots$ and solving for $s_{1}, s_{2}, \ldots$ leads to factors which are determinants of the momenta $p_{i}$. These, however, depend on the integration momentum of the second loop and cannot be pulled out of the tensor integral like in the one-loop case.

In order to show how this technique can successfully be applied to all two-loop selfenergies we first examine, which denominators of tensor integrals can occur after the steps performed in the previous section. They can be read off from the topologies listed in fig. 2.1. Topology 1 clearly is not possible, since at least one of its five different propagators can always be canceled. The topologies 9 through 20 are just products of one-loop contributions. For these the Passarino-Veltman technique can be applied in a straightforward way. The tensor decomposition for the topologies 6 through 8 , having no dependence on the external momentum $p$, is also trivial.

We therefore have to consider the topologies $2-5$. As shown in fig. 4.1 they can be written as $\Delta_{23455}, \Delta_{234}$ and $\Delta_{2345}$, respectively. Note that the scalar integrals corresponding to topologies 4 and 5 are equivalent due to the symmetry relations (3.5). The topologies we have to deal with all contain at least one subloop which is a self-energy insertion, in our terminology this is $\Delta_{23}$. We do the decomposition for this subloop. In the simplest case of a first-rank tensor we have

$$
\left\langle k_{2}^{\mu} \Delta_{23}\right\rangle=k_{5}^{\mu} s\left(k_{5}^{2}\right)
$$

which is just the example considered in (4.4). $k_{5}$ is the external momentum of the subloop $\Delta_{23}$. The Lorentz-scalar $s\left(k_{5}^{2}\right)$ is obtained from

$$
s\left(k_{5}^{2}\right)=\frac{1}{k_{5}^{2}}\left\langle\left(k_{5} \cdot k_{2}\right) \Delta_{23}\right\rangle=\left\langle\left(k_{5} \cdot k_{2}\right) \Delta_{235^{\prime}}\right\rangle .
$$

Due to the self-energy structure of $\Delta_{23}$ the determinant $1 / k_{5}^{2}$ has the form of a massless propagator. Insertion of (4.8) into (4.7) and (4.4) therefore leads to integrals which still belong to the class of $T$ - and $Y$-integrals. We get

$$
p_{\mu} S_{2345}^{2, \mu}=\left\langle\left\langle\left(p \cdot k_{5}\right)\left(k_{5} \cdot k_{2}\right) \Delta_{23455^{\prime}}\right\rangle\right\rangle \text {. }
$$

Since the momenta $k_{5}$ and $k_{2}$ belong to a three vertex in the topology $\Delta_{2345}$ their scalar product is expressible as a sum of squared momenta which can be canceled:

$$
\left(k_{5} \cdot k_{2}\right)=\frac{1}{2}\left(k_{2}^{2}-k_{3}^{2}+k_{5}^{2}\right) \text {. }
$$

The same holds for $\left(p \cdot k_{5}\right)$

$$
\left(p \cdot k_{5}\right)=\frac{1}{2}\left(k_{5}^{2}-k_{4}^{2}+p^{2}\right)
$$

if one notes that one of the $k_{5}^{2}$-terms can be canceled against the massless propagator $\Delta_{5^{\prime}}$. 


\section{$\Delta_{23455} \quad \Delta_{234} \quad \Delta_{2345}$}

Figure 4.1: The topologies representing the denominators of tensor integrals for which the Passarino-Veltman technique is not directly applicable

We can therefore express $Y_{2345}^{1}$ solely in terms of $T$-integrals. The result reads

$$
\begin{aligned}
Y_{2345}^{1}= & A_{0}\left(m_{3}^{2}\right) B_{0}\left(p^{2} ; m_{4}^{2}, m_{5}^{2}\right)-\frac{1}{2}\left\{[ A _ { 0 } ( m _ { 2 } ^ { 2 } ) - A _ { 0 } ( m _ { 3 } ^ { 2 } ) ] \left[\frac{1}{m_{5}^{2}} A_{0}\left(m_{5}^{2}\right)\right.\right. \\
& \left.-B_{0}\left(p^{2} ; m_{4}^{2}, m_{5}^{2}\right)\right]+T_{234}-T_{235}-\left(m_{2}^{2}+m_{3}^{2}+m_{4}^{2}-m_{5}^{2}+p^{2}\right) T_{2345} \\
& -\left(m_{2}^{2}-m_{3}^{2}\right) T_{2355^{\prime}}+\left(m_{4}^{2}-p^{2}\right)\left[T_{2455^{\prime}}-T_{3455^{\prime}}\right] \\
& \left.-\left(m_{2}^{2}-m_{3}^{2}\right)\left(m_{4}^{2}-p^{2}\right) T_{23455^{\prime}}\right\} .
\end{aligned}
$$

The one-loop scalar integrals $A_{0}$ and $B_{0}$ appear since some $T$-integrals are expressible as products of one-loop integrals, e.g.

$$
T_{345}=\left\langle\left\langle\Delta_{345}\right\rangle\right\rangle=\left\langle\Delta_{3}\right\rangle\left\langle\Delta_{45}\right\rangle=A_{0}\left(m_{3}^{2}\right) B_{0}\left(p^{2} ; m_{4}^{2}, m_{5}^{2}\right),
$$

where

$$
\begin{gathered}
A_{0}\left(m^{2}\right)=\left\langle\frac{1}{q^{2}-m^{2}}\right\rangle \\
B_{0}\left(p^{2} ; m_{1}^{2}, m_{2}^{2}\right)=\left\langle\frac{1}{\left[q^{2}-m_{1}^{2}\right]\left[(q+p)^{2}-m_{2}^{2}\right]}\right\rangle
\end{gathered}
$$

and we use

$$
\langle\ldots\rangle=\int \frac{d^{D} q}{i \pi^{2}(2 \pi \mu)^{D-4}}(\ldots)
$$

in analogy to (2.12). Note that our definitions slightly differ from those used in [8].

The integrals containing a massive and a massless propagator with the same momentum can further be simplified by partial fractioning.

The topology $\Delta_{23455}$ is treated in analogy to $\Delta_{2345}$. The corresponding integrals are easily obtained by taking the derivative with respect to $m_{5}^{2}$, e.g.

$$
Y_{23455}^{1}=\frac{\partial}{\partial\left(m_{5}^{2}\right)} Y_{2345}^{1}
$$

The decomposition for the tensors of higher rank can be worked out in a similar manner as described above. In order to treat $Y_{2345}^{11}$ we have to decompose the integral

$$
\left\langle\left\langle\left(p \cdot k_{2}\right)\left(p \cdot k_{2}\right) \Delta_{2345}\right\rangle\right\rangle=p_{\mu} p_{\nu}\left\langle\Delta_{45}\left\langle k_{2}^{\mu} k_{2}^{\nu} \Delta_{23}\right\rangle\right\rangle .
$$


We write

$$
\left\langle k_{2}^{\mu} k_{2}^{\nu} \Delta_{23}\right\rangle=s_{00}\left(k_{5}^{2}\right) g^{\mu \nu}+s_{11}\left(k_{5}^{2}\right) \frac{k_{5}^{\mu} k_{5}^{\nu}}{k_{5}^{2}}
$$

with the Lorentz-scalars $s_{00}\left(k_{5}^{2}\right)$ and $s_{11}\left(k_{5}^{2}\right)$. Contraction with $g_{\mu \nu}$ and $k_{5 \mu} k_{5 \nu}$, respectively, leads to two equations for the scalars $s_{00}$ and $s_{11}$ :

$$
\begin{aligned}
s_{00} D+s_{11} & =\left\langle k_{2}^{2} \Delta_{23}\right\rangle \\
s_{00}+s_{11} & =\frac{1}{k_{5}^{2}}\left\langle\left(k_{5} \cdot k_{2}\right)\left(k_{5} \cdot k_{2}\right) \Delta_{23}\right\rangle .
\end{aligned}
$$

Again, the factors $1 / k_{5}^{2}$ appearing in $(4.19)$ and $(4.20$ can be written as propagators with mass zero. Inspection of (4.20) and (4.18) shows that the decomposition for the integral (4.18) leads to $T$-integrals and one $Y$-integral $Y_{234}^{5}$ which corresponds to a tensor of lower rank. The tensor reduction of this integral will be worked out below, where we discuss the integrals with the topology $\Delta_{234}$. The complete reduction formula for $Y_{2345}^{11}$ is rather lengthy. We give it in the appendix.

The generalization to tensors of higher than second rank should now be obvious. The decomposition of the tensor integrals always leads directly to $T$-integrals or to tensor integrals of lower rank.

So far we showed that the tensor decomposition works for all tensor integrals with the topologies $\Delta_{2345}$ and $\Delta_{23455}$. The third topology we have to consider is $\Delta_{234}$. This case is slightly more complicated due to the presence of the two four-vertices in the topology. The $Y$-integrals which can occur are $Y_{234}^{1}, Y_{234}^{5}, Y_{234}^{11}, Y_{234}^{15}, Y_{234}^{55}, \ldots$ The integrals $Y_{234}^{1}$ and $Y_{234}^{5}$ are not independent of each other but are related through a symmetry relation which amounts to a permutation of the masses $m_{2}$ and $m_{4}$. The same holds, of course, for $Y_{234}^{11}$ and $Y_{234}^{55}$.

We begin with the integral $Y_{234}^{1}$ and apply exactly the same steps as for $Y_{2345}^{1}$. This gives

$$
\begin{aligned}
Y_{234}^{1} & =T_{34}+\left(m_{2}^{2}+p^{2}\right) T_{234}-2 p_{\mu}\left\langle\Delta_{4}\left\langle k_{2}^{\mu} \Delta_{23}\right\rangle\right\rangle \\
& =T_{34}+\left(m_{2}^{2}+p^{2}\right) T_{234}-2\left\langle\left\langle\left(p \cdot k_{5}\right)\left(k_{5} \cdot k_{2}\right) \Delta_{2345^{\prime}}\right\rangle\right\rangle,
\end{aligned}
$$

where (4.7) and (4.8) were used. The last integral looks very similar to (4.9) but here it is not possible to cancel the $k_{5}^{4}$ term resulting from the insertion of (4.10) and (4.11). Instead we obtain

$$
Y_{234}^{1}\left(m_{2}^{2}, m_{3}^{2}, m_{4}^{2}\right)=-\frac{1}{2} Y_{234}^{5}\left(m_{2}^{2}, m_{3}^{2}, m_{4}^{2}\right)+f(T)
$$

where $f(T)$ summarizes terms in which only $T$-integrals occur. In order to find an expression for $Y_{234}^{1}$ in terms of $T$-integrals only, we use the last symmetry relation listed in (3.5) and obtain after appropriate relabeling of the masses

$$
Y_{234}^{5}\left(m_{2}^{2}, m_{3}^{2}, m_{4}^{2}\right)=-\frac{1}{2} Y_{234}^{1}\left(m_{2}^{2}, m_{3}^{2}, m_{4}^{2}\right)+g(T)
$$

where $g(T)$ again depends on $T$-integrals only. Insertion of this equation into (4.22) gives the result

$$
Y_{234}^{1}=\frac{1}{3}\left\{h\left(A_{0}, B_{0}\right)+\left(m_{2}^{2}+m_{3}^{2}+m_{4}^{2}+p^{2}\right) T_{234}+2\left(m_{2}^{2}-m_{3}^{2}\right) T_{235^{\prime}}+\left(m_{3}^{2}-m_{4}^{2}\right) T_{1^{\prime} 34}\right.
$$




$$
\left.+2\left(m_{2}^{2}-m_{3}^{2}\right)\left(m_{4}^{2}-p^{2}\right) T_{2345^{\prime}}+\left(m_{2}^{2}-p^{2}\right)\left(m_{3}^{2}-m_{4}^{2}\right) T_{1^{\prime} 234}\right\},
$$

where $h\left(A_{0}, B_{0}\right)$ represents a function containing only one-loop integrals.

Another complication can be seen by inspecting (4.24). Due to the absence of the momentum $k_{5}$ the integral $Y_{234}^{1}$ is symmetric with respect to the permutation of the momenta $k_{3}$ and $k_{4}$ or, equivalently, of the masses $m_{3}$ and $m_{4}$. However, the tensor decomposition artificially introduces the momentum $k_{5}$ into the terms on the right hand side of (4.24) and the symmetry seems to be lost. It can be made manifest again by using the relation (5.3) stated below. The result for $Y_{234}^{1}$ finally reads

$$
\begin{aligned}
Y_{234}^{1}= & \frac{1}{3}\left\{A_{0}\left(m_{2}^{2}\right) A_{0}\left(m_{3}^{2}\right)+A_{0}\left(m_{2}^{2}\right) A_{0}\left(m_{4}^{2}\right)+A_{0}\left(m_{3}^{2}\right) A_{0}\left(m_{4}^{2}\right)+\left(m_{2}^{2}+m_{3}^{2}+m_{4}^{2}+p^{2}\right) T_{234}\right. \\
& -\left(m_{4}^{2}-p^{2}\right)\left[A_{0}\left(m_{2}^{2}\right)-A_{0}\left(m_{3}^{2}\right)\right] B_{0}\left(p^{2} ; m_{4}^{2}, 0\right)-\left(m_{3}^{2}-p^{2}\right)\left[A_{0}\left(m_{2}^{2}\right)-A_{0}\left(m_{4}^{2}\right)\right] \\
& \times B_{0}\left(p^{2} ; m_{3}^{2}, 0\right)+\left(m_{2}^{2}-m_{3}^{2}\right) T_{235^{\prime}}+\left(m_{2}^{2}-m_{4}^{2}\right) T_{235^{\prime}}\left(m_{2}^{2}, m_{4}^{2}\right) \\
& \left.+\left(m_{2}^{2}-m_{3}^{2}\right)\left(m_{4}^{2}-p^{2}\right) T_{2345^{\prime}}+\left(m_{3}^{2}-p^{2}\right)\left(m_{2}^{2}-m_{4}^{2}\right) T_{2345^{\prime}}\left(m_{2}^{2}, m_{4}^{2}, m_{3}^{2}\right)\right\}
\end{aligned}
$$

which displays the considered symmetry.

The integrals involving higher tensors are treated in exactly the same way. For the second-rank tensor we use (4.19). The reduction of $Y_{234}^{11}$ leads to a formula involving $Y_{234}^{15}$. For this an expression analogous to (4.23) is obtained. The symmetry properties of the integral become manifest after using a relation involving $T$-integrals. The expressions for $Y_{234}^{11}$ and $Y_{234}^{15}$ are listed in the appendix.

With the technique for the tensor decomposition of two-loop tensor integrals described above all $Y$-integrals can be reduced to scalar integrals of simpler structure where no integration momentum appears in the numerator, i.e. to $T$-integrals. The class of $T$ integrals therefore suffices to express all possible two-loop self-energies.

This is true in every renormalizable gauge. For practical purposes we briefly examine the situation in the 't Hooft-Feynman gauge. As explained above the most complicated $Y$-integrals have denominators of the form shown in fig. 4.1. The possible numerators can be obtained by power counting. For $\xi_{i}=1$ the gauge boson propagators (2.11) do not contribute powers of momenta to the numerator of the Feynman integrals. Counting the powers arising from the other Feynman rules and from the decompositions (2.2), (2.4), (2.6) and (2.7) reveals that the relevant integrals are $Y_{234}^{1}, Y_{2345}^{1}, Y_{2345}^{11}, Y_{23455}^{1}$ and $Y_{23455}^{11}$. Explicit formulae for these integrals are given in this section and in the appendix where we also list some reduction formulae needed for general values of $\xi_{i}$.

By using the methods outlined above the task of evaluating each Feynman amplitude corresponding to a two-loop self-energy is reduced to the calculation of a relatively small number of scalar integrals possessing a simpler structure. This is very desirable since a direct evaluation of the tensor integrals appearing in the Feynman amplitude by means of Feynman parameters in general yields a large number of different integrals which all have to be treated separately. In contrast to that the $T$-integrals are well suited for studying their analytical properties as was already done by a number of authors.

In [13, 14, 15, 16], for example, results in terms of polylogarithmic functions were obtained for special cases of masses and momenta. In more general cases this class of 
functions is not sufficient. As was shown in [17], the general massive two-loop integrals cannot be expressed in terms of Nielsen polylogarithms with arguments being algebraic functions of the external variables. For the "master integral" (fig. 2.2) an integral representation suitable for numerical evaluation was derived in [18].

\section{Relations between the scalar integrals}

The results obtained for the two-loop self-energies via the steps described in the preceding sections consist of a Lorentz tensor specified in (2.1), (2.3) and (2.5), respectively, and a scalar part being a sum of $T$-integrals multiplied by rational functions in the squared momentum $p^{2}$, the space-time dimension $D$, the particle masses $m_{j}$ and the gauge parameters $\xi_{i}$. The algebraic structure of the result is convenient for studying the dependence on these variables, either by inserting the exact results for the integrals, if these are known, or suitable approximations, e.g. in the asymptotic or threshold regime of $p^{2}$ or in the limit $D \rightarrow 4$.

The $T$-integrals occurring in the result, which we denote as $T^{1}, T^{2}, \ldots, T^{n}$ for the moment, are in general not algebraically independent of each other, i.e. there exist relations

$$
c^{1} T^{1}+c^{2} T^{2}+\ldots+c^{n} T^{n}=0 .
$$

The coefficients $c^{1}, \ldots, c^{n}$ are polynomials in $p^{2}, D, m_{j}$ and the gauge parameters. The results can be made very transparent by using these relations to eliminate as many integrals as possible. For example, if one considers a gauge invariant set of amplitudes this property directly manifests itself in the algebraic result provided that the set of integrals used for expressing the result is sufficiently small. In this case all terms depending on the gauge parameters $\xi_{i}$ disappear from the result. This means that the prefactors of all gauge dependent basic integrals algebraically add up to zero while all $\xi_{i}$-dependent terms multiplying a gauge independent basic integral exactly cancel each other. In this way the gauge invariance is seen to hold exactly, i.e. by purely algebraical means. No explicit analytical or numerical expressions of the standard integrals are needed for this consideration.

In the same way as the gauge invariance Slavnov-Taylor identities or any other relation involving two-loop self-energies can be checked exactly. This is very useful for performing consistency checks on the result.

The question arises whether a sufficiently small basis of integrals can actually be found. We argue that after invoking the symmetry properties of the two-loop integrals and eliminating all $Y$-integrals by means of tensor decompositions only few additional relations between the scalar integrals are needed. In sect. 7 we will verify Slavnov-Taylor identities valid for the self-energies of the photon and the $Z$-boson by adding up the results of several thousand Feynman amplitudes. For this application only the relations for the integrals $T_{2345^{\prime}}\left(m_{2}^{2}, m_{3}^{2}, m_{4}^{2}\right)$ and $T_{2345^{\prime} 5^{\prime}}\left(m_{2}^{2}, m_{3}^{2}, m_{4}^{2}\right)$ described below are used.

The first type of relations we want to consider involves integrals in which at least one propagator is massless. They are obtained indirectly by using symmetry arguments, momentum conservation and properties of the tensor decomposition. In this way we

derive a relation between the integral $T_{2345^{\prime}}\left(m_{2}^{2}, m_{3}^{2}, m_{4}^{2}\right)$ and the integrals of the same 
type where the masses are permuted. It is a consequence of momentum conservation at the four-vertices of the topology $\Delta_{234}$. Starting from

$$
\left\langle\left\langle\frac{p \cdot\left(k_{2}+k_{3}-k_{4}-p\right)}{\left[k_{2}^{2}-m_{2}^{2}\right]\left[k_{3}^{2}-m_{3}^{2}\right]\left[k_{4}^{2}-m_{4}^{2}\right]}\right\rangle\right\rangle=0,
$$

which obviously follows from (2.17), using the symmetries of the resulting integrals with respect to permutations of $k_{2}, k_{3}$ and $k_{4}$ and performing tensor decompositions for these leads to the relation

$$
\begin{aligned}
& \left\{\left(m_{2}^{2}-m_{3}^{2}\right)\left(m_{4}^{2}-p^{2}\right) T_{2345^{\prime}}\left(m_{2}^{2}, m_{3}^{2}, m_{4}^{2}\right)+\text { cycl. }\right\} \\
& +\left\{\left(m_{2}^{2}-m_{3}^{2}\right) T_{235^{\prime}}\left(m_{2}^{2}, m_{3}^{2}\right)+\text { cycl. }\right\} \\
& -\left\{\left[A_{0}\left(m_{2}^{2}\right)-A_{0}\left(m_{3}^{2}\right)\right]\left(m_{4}^{2}-p^{2}\right) B_{0}\left(p^{2} ; m_{4}^{2}, 0\right)+\text { cycl. }\right\}=0 .
\end{aligned}
$$

Here "cycl." denotes cyclic permutation of the masses. With (5.3) one permutation of the $T_{2345^{\prime}}$-integral can always be eliminated. This relation was used in the last section to obtain (4.25). If two masses are equal, (5.3) becomes trivial.

Two similar relations hold for the permutations of the integral $T_{2345^{\prime} 5^{\prime}}$. Using these, it is possible to obtain one standard permutation for every integral of this type. In contrast to (5.3) this relation remains nontrivial if two of the three masses are equal. It gives an expression for the integral $T_{2345^{\prime} 5^{\prime}}\left(m^{2}, M^{2}, M^{2}\right)$ in terms of integrals with fewer propagators only. Therefore it can completely be replaced by integrals of a simpler type. We list this relation in the appendix. It takes a particularly simple form in the special case where $M^{2}=0$ :

$$
\begin{aligned}
T_{23^{\prime} 4^{\prime} 5^{\prime} 5^{\prime}}\left(m^{2}\right)= & \frac{1}{D m^{2} p^{2}}\left\{A_{0}\left(m^{2}\right)\left[(D-4) B_{0}\left(p^{2} ; 0,0\right)+D p^{2} B_{0}^{\prime}\left(p^{2} ; 0,0\right)\right]\right. \\
& -(D-2)^{2} T_{23^{\prime} 5^{\prime}}\left(m^{2}\right)+(3 D-8) T_{23^{\prime} 4^{\prime}}\left(m^{2}\right) \\
& \left.-(D-4)\left(m^{2}+p^{2}\right) T_{23^{\prime} 4^{\prime} 5^{\prime}}\left(m^{2}\right)\right\} .
\end{aligned}
$$

The one-loop integral $B_{0}^{\prime}$ is defined as

$$
B_{0}^{\prime}\left(p^{2} ; m_{1}^{2}, m_{2}^{2}\right)=\frac{\partial}{\partial\left(m_{1}^{2}\right)} B_{0}\left(p^{2} ; m_{1}^{2}, m_{2}^{2}\right)
$$

Relations similar to those valid for $T_{2345^{\prime}}$ and $T_{2345^{\prime} 5^{\prime}}$ can be obtained for integrals with more massless propagators, e.g. $T_{2345^{\prime} 5^{\prime} 5^{\prime}}$ and $T_{2345^{\prime} 5^{\prime} 5^{\prime} 5^{\prime}}$.

A second class of relations can be derived via the well known method of integration by parts. It yields relations for integrals where at least one propagator appears with a power higher than one. A particularly interesting example is the formula for the integral corresponding to topology 6 in fig. 2.1, i.e. $T_{1134}\left(m_{1}^{2}, m_{1}^{2}, m_{3}^{2}, m_{4}^{2}\right)$. Starting from

$$
D T_{134}=\left\langle\left\langle\Delta_{134} \frac{\partial k_{1}^{\mu}}{\partial k_{1}^{\mu}}\right\rangle\right\rangle=-\left\langle\left\langle k_{1}^{\mu} \frac{\partial}{\partial k_{1}^{\mu}} \Delta_{134}\right\rangle\right\rangle
$$


and using the symmetry of the integral $T_{134}$ with respect to permutation of the masses $m_{1}^{2}$ and $m_{3}^{2}$ leads to

$$
\begin{aligned}
T_{1134}= & \frac{-1}{\lambda\left(m_{1}^{2}, m_{3}^{2}, m_{4}^{2}\right)}\left\{(3-D)\left(m_{1}^{2}-m_{3}^{2}-m_{4}^{2}\right) T_{134}-(1-D / 2)\left[A _ { 0 } ( m _ { 1 } ^ { 2 } ) \left(A_{0}\left(m_{3}^{2}\right)\right.\right.\right. \\
& \left.\left.\left.+A_{0}\left(m_{4}^{2}\right)\right)-2 A_{0}\left(m_{3}^{2}\right) A_{0}\left(m_{4}^{2}\right)\right]-\left(m_{3}^{2}-m_{4}^{2}\right)\left(A_{0}\left(m_{3}^{2}\right)-A_{0}\left(m_{4}^{2}\right)\right) B_{0}\left(0 ; m_{1}^{2}, m_{1}^{2}\right)\right\}
\end{aligned}
$$

where

$$
\lambda\left(m_{1}^{2}, m_{3}^{2}, m_{4}^{2}\right)=m_{1}^{4}+m_{3}^{4}+m_{4}^{4}-2\left(m_{1}^{2} m_{3}^{2}+m_{1}^{2} m_{4}^{2}+m_{3}^{2} m_{4}^{2}\right) .
$$

The integral $T_{1134}$ can therefore be expressed through $T_{134}$ and products of one-loop integrals.

Finally it should be noted that some integrals vanish trivially in the framework of dimensional regularization, e.g.

$$
T_{11}=T_{12}=T_{112}=0
$$

\section{Computer-algebraic realization}

The procedure we described for the algebraic calculation of two-loop self-energies is algorithmic and we implemented it into a computer-algebra program which we called Two Calc [12]. It is written in Mathematica and linked to the packages FeynArts [19] and FeynCalc [20]. FeynArts creates the Feynman amplitudes and draws the Feynman graphs. FeynCalc is used here for the contraction of Lorentz indices and the evaluation of Dirac-traces. In the calculations performed in this paper an anticommuting $\gamma_{5}$ in $D$ dimensions was used.

The three packages run fully automatically. This high degree of automation is very convenient for performing calculations which involve a large number of diagrams.

\section{Slavnov-Taylor identities for two-loop self-energies}

In order to demonstrate the abilities of our algorithm for doing large calculations we explicitly verify some Slavnov-Taylor identities valid for two-loop self-energies. In this section we use the 't Hooft-Feynman gauge.

In [21] exact relations for the gauge boson propagators $\Delta_{\mu \nu}^{\alpha \beta}$, the gauge boson Higgs mixing propagators $\Delta_{\mu}^{\alpha i}$ and the unphysical Higgs propagators $\Delta^{i j}$ are listed

$$
\begin{array}{ll}
p^{\mu} p^{\nu} \Delta_{\mu \nu}^{\gamma \gamma}(p) & =-i \\
p^{\mu} p^{\nu} \Delta_{\mu \nu}^{Z Z}(p)-2 i M_{Z} p^{\mu} \Delta_{\mu}^{Z \chi}(p)+M_{Z}^{2} \Delta^{\chi \chi}(p) & =-i \\
p^{\mu} p^{\nu} \Delta_{\mu \nu}^{\gamma Z}(p)-i M_{Z} p^{\mu} \Delta_{\mu}^{\gamma \chi}(p) & =0 \\
p^{\mu} p^{\nu} \Delta_{\mu \nu}^{W W}(p)+2 M_{W} p^{\mu} \Delta_{\mu}^{W \varphi}(p)+M_{W}^{2} \Delta^{\varphi \varphi}(p) & =-i
\end{array}
$$


where $\chi$ and $\varphi$ are the neutral and charged unphysical Higgs fields, respectively. Writing the propagators as the inverse of the truncated one-particle irreducible two-point functions as specified in sect. 2 and expanding up to second order leads to the second-order relations

$$
\begin{array}{ll}
p^{2}\left[\Sigma_{L}^{\gamma \gamma,(2)}-\left(\Sigma^{\gamma \chi,(1)}\right)^{2}\right] & =0 \\
p^{2}\left[\Sigma_{L}^{Z Z,(2)}-2 i M_{Z} \Sigma^{Z \chi,(2)}\right]-M_{Z}^{2} \Sigma^{\chi \chi,(2)}-p^{2}\left(\Sigma^{Z \chi,(1)}\right)^{2}+\Sigma_{L}^{Z Z,(1)} \Sigma^{\chi \chi,(1)} & =0 \\
p^{2}\left[\Sigma_{L}^{\gamma Z,(2)}-i M_{Z} \Sigma^{\gamma \chi,(2)}\right]-p^{2} \Sigma^{\gamma \chi,(1)} \Sigma^{Z \chi,(1)}+i M_{Z} \Sigma^{\gamma \chi,(1)} \Sigma^{\chi \chi,(1)} & =0 \\
p^{2}\left[\Sigma_{L}^{W W,(2)}+2 M_{W} \Sigma^{W \varphi,(2)}\right]-M_{W}^{2} \Sigma^{\varphi \varphi,(2)}-p^{2}\left(\Sigma^{W \varphi,(1)}\right)^{2}+\Sigma_{L}^{W W,(1)} \Sigma^{\varphi \varphi,(1)} & =0 .
\end{array}
$$

The superscripts ${ }^{(n)}$ indicate the two-loop and one-loop self-energies, respectively. Similar relations can be obtained for the one-loop self-energies [21]. As always, it is understood that the tadpole contributions are part of the self-energies (see fig. 2.1).

The first relation in (7.2) indicates that unlike in pure QED the longitudinal part of the two-loop photon self-energy does not vanish. We used TwoCalc to calculate explicitly every Feynman amplitude contributing to the left hand side of this relation. We did this in the full electroweak SM with the only restriction that the fermions are limited to one doublet, which we chose to be the $u$ - and $d$-quark. The calculations for all other fermions can be performed analogously.

We had to consider 1024 graphs for the two-loop photon self-energy, 162 of which contain the $u$ - and $d$-quarks, the remaining ones are purely bosonic. The most involved diagrams are of course those stemming from the generic two-loop topologies. These are 418 diagrams belonging to the first 6 topologies in fig. 2.1. The remaining ones lead to integrals expressible solely as products of one-loop integrals. Altogether 13 of the 20 possible topologies are realized for the photon self-energy.

The one-loop $(\gamma \chi)$-mixing self-energy vanishes for fermionic graphs, while 4 purely bosonic graphs contribute. Summing up the results of all amplitudes gives exactly zero, i.e. the prefactor of every standard integral vanishes separately.

We also explicitly verified the second relation stated in (7.2). Here, of course, a lot more Feynman diagrams have to be calculated. Again we restricted the fermions to $u$ -

and $d$-quark. This gives 1552 diagrams for $\Sigma_{L}^{Z Z,(2)}, 1155$ for $\Sigma^{Z \chi,(2)}$ and 1142 for $\Sigma^{\chi \chi,(2)}$. From these, 1787 diagrams arise from the 8 generic two-loop topologies.

For the self-energies of the $Z$-boson and the unphysical Higgs field $\chi$ all 20 topologies contribute which are maximally possible for two-loop self-energies. The self-energies in one-loop order give rise to 65 diagrams. Adding those to the 3849 two-loop graphs by using the coefficients specified in (7.2) gives exactly zero.

\section{Results for the electroweak Standard Model and discussion}

As an application of the techniques described above we treat the gauge boson selfenergies in the electroweak SM. We begin with the special case of the photon self-energy in pure QED. The three contributing diagrams are shown in fig. 8.1. 
Figure 8.1: The diagrams contributing to the two-loop photon self-energy in QED.

We decompose the photon self-energy according to (2.1). The longitudinal part vanishes due to the well-known Ward-identity. We checked this by explicit calculation. The result for the transverse part reads

$$
\begin{aligned}
\Sigma_{T, Q E D}^{\gamma \gamma,(2)}\left(p^{2}\right)= & \frac{e^{4}}{128 \pi^{4}(D-1)}\left\{\left[(2-D)^{3} / m_{e}^{2}\right] A_{0}^{2}\left(m_{e}^{2}\right)-\left[(2-D)^{2}\left(m_{e}^{2}-p^{2}\right) / m_{e}^{2}\right]\right. \\
& \times A_{0}\left(m_{e}^{2}\right) B_{0}\left(p^{2} ; 0, m_{e}^{2}\right)-\left[(2-D)\left(4 m_{e}^{2}+2 p^{2}-D p^{2}\right) / m_{e}^{2}\right] A_{0}\left(m_{e}^{2}\right) \\
& \times B_{0}\left(p^{2} ; m_{e}^{2}, m_{e}^{2}\right)-\left(8 m_{e}^{2}-14 p^{2}+9 D p^{2}-D^{2} p^{2}\right) B_{0}^{2}\left(p^{2} ; m_{e}^{2}, m_{e}^{2}\right) \\
& -(2-D)\left[2\left(4 m_{e}^{2}-2 p^{2}+D p^{2}\right) A_{0}\left(m_{e}^{2}\right) B_{0}^{\prime}\left(p^{2} ; m_{e}^{2}, m_{e}^{2}\right)+(2-D) T_{13^{\prime} 4^{\prime}}\right. \\
& \left.-2(1-D) T_{134^{\prime}}-(6-D) T_{234^{\prime}}+(2-D)\left(m_{e}^{2}-p^{2}\right) T_{1^{\prime} 234^{\prime}}\right] \\
& +8\left(4 m_{e}^{2}-2 p^{2}+D p^{2}\right)\left[T_{1234^{\prime}}+m_{e}^{2} T_{11234^{\prime}}\right] \\
& \left.+2\left(2 m_{e}^{2}-p^{2}\right)\left(4 m_{e}^{2}-2 p^{2}+D p^{2}\right) T_{123^{\prime} 45}\right\}
\end{aligned}
$$

As above a prime at the subindices of the $T$-integrals denotes that the corresponding propagator has mass zero. All other propagators carry the electron mass $m_{e}$.

The calculation was carried out in an arbitrary $R_{\xi}$-gauge. The absence of the gauge parameter $\xi_{\gamma}$ indicates that the result is gauge invariant as it has to be. The two-loop integrals appearing in (8.1) are expressible in terms of polylogarithmic functions, i.e. logarithms, di- and trilogarithms. They were studied for example in [15, 16]. The one-loop integrals arising from the calculation of irreducible two-loop contributions are in general needed up to $\mathcal{O}(D-4)$. Results for these can be found in [13, 22].

Calculations of the two-loop photon self-energy in QED were performed in 23]. Similar calculations focusing on the $\mathcal{O}\left(\alpha \alpha_{s}\right)$ corrections due to the gluon exchange in a quark loop of gauge boson self-energies were done in [24].

In passing we note that in contrast to the photon self-energy the two-loop electron self-energy in QED is not gauge invariant. The explicit algebraic result will be presented elsewhere. It involves the integrals $T_{234}, T_{1^{\prime} 234}$ and $T_{1^{\prime} 2345^{\prime}}$ which cannot be reduced to polylogarithmic functions. The problem of evaluating $T_{1^{\prime} 2345^{\prime}}$ was addressed in [15].

In the electroweak SM results for two-loop gauge boson self-energies restricted to the region $p^{2}=0$ and asymptotic values of the top and Higgs mass, respectively, were worked out in [3], 4, 5]. A calculation allowing for general values of the invariant momentum $p^{2}$ and the masses of the gauge bosons, the top quark and the Higgs field has to deal with an enormous number of Feynman diagrams having in general a very complicated structure. 
With the methods outlined above we can express these in terms of a small number of standard integrals, the result being valid for general values of all parameters involved.

This was explicitly carried out in sect. 7 for all graphs contributing to the longitudinal parts of the photon and $Z$-boson self-energies. The results for the complete transverse parts are of course rather lengthy and we do not present them here. Instead we focus on the light fermion contributions, a class of diagrams particularly well suited for separate treatment which is expected to yield a substantial contribution to the complete result.

With light fermion contributions we mean all diagrams containing any fermion other than the top-quark. These fermion masses are negligible in comparison to the boson masses $\left(m_{Z}, m_{W}, m_{H}\right)$ and the invariant momentum $p^{2}$, if $p^{2}$ is sufficiently large. All $T$ integrals resulting from these graphs therefore contain one massless fermion loop. This feature allows an analytic evaluation resulting in polylogarithmic functions. We have explicitly calculated all integrals needed for the two-loop gauge boson self-energies, i.e. all integrals appearing in the algebraic results stated below. The results will be presented in a related paper [13].

In order to study the structure of the results we find it sufficient to restrict the fermions to one doublet in the same way as in sect. 7. This yields 27 diagrams contributing to the two-loop photon self-energy, 32 graphs for the $Z$ self-energy and 45 diagrams for the self-energy of the $W^{ \pm}$.

As shown in fig. 8.2 it is convenient to subdivide the photon self-energy into several sets of graphs. The graphs depicted in fig. 8.2 a are of QED-type having a photon exchanged in a quark loop. As indicated in the picture the same set of graphs also exists for the $u$-quark. The results are in exact analogy to (8.1) and we do not write them out explicitly. As a consequence of (8.1) the QED-type graphs form gauge invariant subsets of the photon self-energy.

In the graphs shown in fig. 8.2 b a $Z$-boson is exchanged instead of the $\gamma$. We give the result neglecting the mass of the $d$-quark:

$$
\begin{aligned}
\Sigma_{T, Z}^{\gamma \gamma,(2)}\left(p^{2}\right)= & \frac{e^{4}(2-D)\left(9-12 s_{W}^{2}+8 s_{W}^{4}\right)}{(4 \pi)^{4}(1-D) D 108 c_{W}^{2} s_{W}^{2}}\left\{2\left(4-6 D+D^{2}\right) A_{0}\left(m_{Z}^{2}\right) B_{0}\left(p^{2} ; 0,0\right)\right. \\
& -D\left(2 m_{Z}^{2}+7 p^{2}-D p^{2}\right) B_{0}^{2}\left(p^{2} ; 0,0\right)+2\left(4-6 D+D^{2}\right) T_{13^{\prime} 4^{\prime}}\left(m_{Z}^{2}\right) \\
& +2\left(8-4 D+D^{2}\right) T_{23^{\prime} 4^{\prime}}\left(m_{Z}^{2}\right)-2\left(4-8 D+D^{2}\right)\left(m_{Z}^{2}+p^{2}\right) T_{1^{\prime} 2^{\prime} 34^{\prime}}\left(m_{Z}^{2}\right) \\
& \left.-D\left(2 m_{Z}^{4}+8 m_{Z}^{2} p^{2}-D m_{Z}^{2} p^{2}+2 p^{4}\right) T_{1^{\prime} 2^{\prime} 34^{\prime} 5^{\prime}}\left(m_{Z}^{2}\right)\right\} .
\end{aligned}
$$

The subscript $Z$ refers to the $Z$-boson exchange. We used the abbreviations

$$
c_{W}^{2}=\frac{m_{W}^{2}}{m_{Z}^{2}}, \quad s_{W}^{2}=1-\frac{m_{W}^{2}}{m_{Z}^{2}} .
$$

It can be seen from (8.2) that for non-exceptional values of $p^{2}$ no mass singularities are induced by setting the fermion mass to zero (see [25]).

There is no gauge dependence left in (8.2). Therefore all contributions from "neutral currents", i.e. $\gamma$ and $Z$ exchange, are gauge invariant. 
$d \leftrightarrow u$

$(a)$

$$
d \leftrightarrow u
$$

(b)

$(c)$

Figure 8.2: The light fermion contributions to the two-loop photon self-energy 
The "charged currents" mediated by $W^{ \pm}$and $\varphi^{ \pm}$give rise to the 15 diagrams shown in fig. 8.2 c. The result for the transverse part reads

$$
\begin{aligned}
\Sigma_{T, W^{ \pm}}^{\gamma \gamma,(2)}\left(p^{2}\right)= & \frac{e^{4}(2-D)}{(4 \pi)^{4}(1-D)^{2} 12 m_{W}^{4} s_{W}^{2}}\left\{f\left(p^{2}, m_{W}^{2}, D\right)+9 g\left(\xi_{W}, p^{2}, m_{W}^{2}, D\right)\right. \\
& \left.+F\left(p^{2}, m_{W}^{2}, D\right)+9 m_{W}^{2}\left(2 m_{W}^{2}-p^{2}\right) G\left(\xi_{W}, p^{2}, m_{W}^{2}, D\right)\right\},
\end{aligned}
$$

where in $f\left(p^{2}, m_{W}^{2}, D\right)$ and $g\left(\xi_{W}, p^{2}, m_{W}^{2}, D\right)$ we collected the contributions involving only one-loop integrals

$$
\begin{aligned}
f\left(p^{2}, m_{W}^{2}, D\right)= & 2 m_{W}^{2}\left[\left(\frac{40}{D}-91+70 D-10 D^{2}\right) m_{W}^{2}-18(2-D) p^{2}\right] A_{0}\left(m_{W}^{2}\right) B_{0}\left(p^{2} ; 0,0\right) \\
+ & 8(1-D) m_{W}^{4}\left[2 m_{W}^{2}+(7-D) p^{2}\right] B_{0}^{2}\left(p^{2} ; 0,0\right)-9\left(2 m_{W}^{2}-p^{2}\right) \\
\times[ & \left.4(1-D) m_{W}^{4}+4(3-2 D) m_{W}^{2} p^{2}-p^{4}\right] B_{0}\left(p^{2} ; 0,0\right) B_{0}\left(p^{2} ; m_{W}^{2}, m_{W}^{2}\right), \\
g\left(\xi_{W}, p^{2}, m_{W}^{2}, D\right)= & -\frac{2 m_{W}^{2}}{\xi_{W}}\left(m_{W}^{2}-p^{2}\right) A_{0}\left(m_{W}^{2}\right) B_{0}\left(p^{2} ; 0,0\right)+2 m_{W}^{2}\left[\frac{1}{\xi_{W}}\left(m_{W}^{2}-p^{2}\right)-m_{W}^{2}\right. \\
& \left.+2(2-D) p^{2}\right] A_{0}\left(m_{W}^{2} / \xi_{W}\right) B_{0}\left(p^{2} ; 0,0\right)-2\left(m_{W}^{2}-p^{2}\right)\left[m_{W}^{4}\left(\frac{1}{\xi_{W}}-1\right)^{2}\right. \\
& \left.-2\left(\frac{1}{\xi_{W}}+3-2 D\right) m_{W}^{2} p^{2}+p^{4}\right] B_{0}\left(p^{2} ; 0,0\right) B_{0}\left(p^{2} ; m_{W}^{2}, m_{W}^{2} / \xi_{W}\right) \\
& +p^{4}\left(4 \frac{m_{W}^{2}}{\xi_{W}}-p^{2}\right) B_{0}\left(p^{2} ; 0,0\right) B_{0}\left(p^{2} ; m_{W}^{2} / \xi_{W}, m_{W}^{2} / \xi_{W}\right),
\end{aligned}
$$

and $F\left(p^{2}, m_{W}^{2}, D\right)$ and $G\left(\xi_{W}, p^{2}, m_{W}^{2}, D\right)$ contain the generic two-loop contributions

$$
\begin{aligned}
F\left(p^{2}, m_{W}^{2}, D\right)= & m_{W}^{2}\left[\left[2\left(\frac{40}{D}-118+187 D-100 D^{2}+18 D^{3}\right) m_{W}^{2}-9(7-4 D)(3-D) p^{2}\right]\right. \\
& \times T_{13^{\prime} 4^{\prime}}\left(m_{W}^{2}\right)+\left[4\left(\frac{40}{D}-96+61 D-14 D^{2}\right) m_{W}^{2}+9 p^{2}\right] T_{23^{\prime} 4^{\prime}}\left(m_{W}^{2}\right) \\
& -20(1 / D-1)\left(4-8 D+D^{2}\right) m_{W}^{2}\left(m_{W}^{2}+p^{2}\right) T_{1^{\prime} \prime^{\prime} 34^{\prime}}\left(m_{W}^{2}\right)+18\left(4 m_{W}^{2}\right. \\
& \left.-p^{2}\right)\left[2(1-D) m_{W}^{2}+(3-2 D) p^{2}\right] T_{123^{\prime} 4^{\prime}}\left(m_{W}^{2}, m_{W}^{2}\right)+9\left(4 m_{W}^{2}-p^{2}\right) \\
& \times\left[4(1-D) m_{W}^{4}+4(3-2 D) m_{W}^{2} p^{2}-p^{4}\right] T_{1123^{\prime} 4^{\prime}}\left(m_{W}^{2}, m_{W}^{2}, m_{W}^{2}\right) \\
+ & 72(1-D) m_{W}^{4}\left(m_{W}^{2}+2 p^{2}\right) T_{123^{\prime} 4^{\prime} 5^{\prime}}\left(m_{W}^{2}, m_{W}^{2}\right) \\
+ & \left.8(1-D) m_{W}^{2}\left[2\left(m_{W}^{4}+p^{4}\right)+(8-D) m_{W}^{2} p^{2}\right] T_{1^{\prime} 2^{\prime} 34^{\prime} 5^{\prime}}\left(m_{W}^{2}\right)\right], \\
G\left(\xi_{W}, p^{2}, m_{W}^{2}, D\right)= & \frac{1}{\xi_{W}}(D-3) T_{13^{\prime} 4^{\prime}}\left(m_{W}^{2}\right)+T_{23^{\prime} 4^{\prime}}\left(m_{W}^{2} / \xi_{W}\right)-2\left[\left(\frac{1}{\xi_{W}}-1\right) m_{W}^{2}\right. \\
& \left.+(3-2 D) p^{2}\right] T_{123^{\prime} 4^{\prime}}\left(m_{W}^{2}, m_{W}^{2} / \xi_{W}\right)+\left[m_{W}^{4}\left(\frac{1}{\xi_{W}}-1\right)^{2}\right. \\
& \left.-2\left(\frac{1}{\xi_{W}}+3-2 D\right) m_{W}^{2} p^{2}+p^{4}\right] T_{1123^{\prime} 4^{\prime}}\left(m_{W}^{2}, m_{W}^{2}, m_{W}^{2} / \xi_{W}\right) . \quad(8.8)
\end{aligned}
$$


Figure 8.3: Higgs-dependent contributions to the $Z$ self-energy

The functions $f\left(p^{2}, m_{W}^{2}, D\right)$ and $F\left(p^{2}, m_{W}^{2}, D\right)$ are independent of the gauge parameter $\xi_{W}$. The occurrence of $\xi_{W}$ in $g\left(\xi_{W}, p^{2}, m_{W}^{2}, D\right)$ and $G\left(\xi_{W}, p^{2}, m_{W}^{2}, D\right)$ indicates that the contribution from the "charged current" graphs is gauge dependent. This feature is expected since also in one-loop order the graphs containing $W$-bosons give gauge dependent contributions to the photon self-energy (see for example [11]).

As explained above the integrals occurring in (8.4) can be solved analytically for general values of $\xi_{W}$. Using the expressions given in [13] the dependence on the variables $p^{2}, m_{W}^{2}, D$ and $\xi_{W}$ can be studied.

For all results contributing to the photon self-energy we also calculated the longitudinal part. It vanishes separately for each set of graphs specified above and without restriction on the values of the gauge parameters. We also studied the limit $p^{2} \rightarrow 0$ for all results given above and checked that they give zero for all values of the gauge parameters and the dimension $D$.

Next we focus on the self-energy of the $Z$-boson. We first note that there are contributions from 27 graphs corresponding to the ones considered for the photon self-energy, i.e. from the diagrams shown in fig. 8.2 where the in- and outgoing photon is substituted by a $Z$. In addition to these, five graphs occur containing the Higgs-field, three of which are tadpole graphs. These diagrams are depicted in fig. 8.3.

We begin with the diagrams which are of the same form as in the case of the photon. The transverse parts of the "neutral current" graphs are treated in precisely the same way as described above. The results have the same form and contain exactly the same integrals as the corresponding ones for the photon self-energy. Since we are only interested in the structure of the results we do not list them here explicitly. As above, these graphs yield gauge invariant contributions.

For the 15 "charged current" graphs we obtain:

$$
\begin{aligned}
\Sigma_{T, W^{ \pm}}^{Z Z,(2)}\left(p^{2}\right)= & \frac{e^{4}(2-D)}{(4 \pi)^{4}(1-D)^{2} 12 m_{W}^{2} m_{Z}^{2} s_{W}^{4}}\left\{\tilde{f}\left(p^{2}, m_{W}^{2}, m_{Z}^{2}, D\right)\right. \\
& +9 g\left(\xi_{W}, p^{2}, m_{W}^{2}, D\right)+\tilde{F}\left(p^{2}, m_{W}^{2}, m_{Z}^{2}, D\right) \\
& \left.+9\left(p^{2}-m_{Z}^{2}\right)\left(2 m_{W}^{2}-m_{Z}^{2}-p^{2}\right) \frac{m_{W}^{2}}{p^{2}} G\left(\xi_{W}, p^{2}, m_{W}^{2}, D\right)\right\} .
\end{aligned}
$$

The gauge dependent functions $g\left(\xi_{W}, p^{2}, m_{W}^{2}, D\right)$ and $G\left(\xi_{W}, p^{2}, m_{W}^{2}, D\right)$ are the same as for the photon self-energy. They were given in (8.6) and (8.8), respectively. The expressions for the gauge independent functions $\tilde{f}\left(p^{2}, m_{W}^{2}, m_{Z}^{2}, D\right)$ and $\tilde{F}\left(p^{2}, m_{W}^{2}, m_{Z}^{2}, D\right)$ read

$$
\tilde{f}\left(p^{2}, m_{W}^{2}, m_{Z}^{2}, D\right)=2\left[(1 / D-1)\left(4-6 D+D^{2}\right)\left(9 m_{W}^{4}+\left(m_{W}^{2}-m_{Z}^{2}\right)^{2}\right)+9 m_{W}^{2}\left(m_{W}^{2}\right.\right.
$$




$$
\begin{aligned}
& \left.\left.-2(2-D) p^{2}\right)\right] A_{0}\left(m_{W}^{2}\right) B_{0}\left(p^{2} ; 0,0\right)+(1-D)\left(4 m_{W}^{2}-m_{Z}^{2}\right)\left(2 m_{W}^{2}\right. \\
& \left.+m_{Z}^{2}\right)\left[2 m_{W}^{2}+(7-D) p^{2}\right] B_{0}^{2}\left(p^{2} ; 0,0\right)-9\left(2 m_{W}^{2}-p^{2}\right)\left[4(1-D) m_{W}^{4}\right. \\
& \left.+4(3-2 D) m_{W}^{2} p^{2}-p^{4}\right] B_{0}\left(p^{2} ; 0,0\right) B_{0}\left(p^{2} ; m_{W}^{2}, m_{W}^{2}\right), \\
\tilde{F}\left(p^{2}, m_{W}^{2}, m_{Z}^{2}, D\right)= & {\left[2\left(\frac{40}{D}-118+187 D-100 D^{2}+18 D^{3}\right) m_{W}^{4}-\left(\frac{8}{D}+7+5 D-2 D^{2}\right.\right.} \\
& \left.\left.+9(2-D) \frac{m_{W}^{2}}{p^{2}}\right) m_{Z}^{2}\left(2 m_{W}^{2}-m_{Z}^{2}\right)-9(7-4 D)(3-D) m_{W}^{2} p^{2}\right] \\
& \times T_{13^{\prime} 4^{\prime}}\left(m_{W}^{2}\right)+\left[4\left(\frac{40}{D}-96+61 D-14 D^{2}\right) m_{W}^{4}-2(1 / D-1)\right. \\
& \left.\times\left(8-4 D+D^{2}\right) m_{Z}^{2}\left(2 m_{W}^{2}-m_{Z}^{2}\right)+9 m_{W}^{2} p^{2}\right] T_{23^{\prime} 4^{\prime}}\left(m_{W}^{2}\right) \\
& -2(1 / D-1)\left(4-8 D+D^{2}\right)\left(m_{W}^{2}+p^{2}\right)\left(9 m_{W}^{4}+\left(m_{W}^{2}-m_{Z}^{2}\right)^{2}\right) \\
& \times T_{1^{\prime} 2^{\prime} 34^{\prime}}\left(m_{W}^{2}\right)+18 m_{W}^{2}\left(4 m_{W}^{2}-p^{2}\right)\left[2(1-D) m_{W}^{2}+(3-2 D) p^{2}\right] \\
& \times T_{123^{\prime} 4^{\prime}}\left(m_{W}^{2}, m_{W}^{2}\right)+9 m_{W}^{2}\left(4 m_{W}^{2}-p^{2}\right)\left[4(1-D) m_{W}^{4}\right. \\
& \left.+4(3-2 D) m_{W}^{2} p^{2}-p^{4}\right] T_{1123^{\prime} 4^{\prime}}\left(m_{W}^{2}, m_{W}^{2}, m_{W}^{2}\right) \\
& +72(1-D) m_{W}^{6}\left(m_{W}^{2}+2 p^{2}\right) T_{123^{\prime} 4^{\prime} 5^{\prime}}\left(m_{W}^{2}, m_{W}^{2}\right)+(1-D)\left(4 m_{W}^{2}\right. \\
& \left.-m_{Z}^{2}\right)\left(2 m_{W}^{2}+m_{Z}^{2}\right)\left[2\left(m_{W}^{4}+p^{4}\right)+(8-D) m_{W}^{2} p^{2}\right] T_{1^{\prime} 2^{\prime} 34^{\prime} 5^{\prime}}\left(m_{W}^{2}\right) . \\
&
\end{aligned}
$$

For $m_{Z}^{2}=0$ the functions $\tilde{f}\left(p^{2}, m_{W}^{2}, m_{Z}^{2}, D\right)$ and $\tilde{F}\left(p^{2}, m_{W}^{2}, m_{Z}^{2}, D\right)$ coincide with the corresponding ones used for the photon self-energy, i.e. $f\left(p^{2}, m_{W}^{2}, D\right)$ and $F\left(p^{2}, m_{W}^{2}, D\right)$.

The characteristic feature of the result (3.9) is that all gauge dependent contributions involving two-loop integrals are proportional to $\left(p^{2}-m_{Z}^{2}\right)$. This can be understood either by noting that $p^{2}=m_{Z}^{2}$ is the mass shell condition or, in the framework of the intrinsic pinch technique [10], by observing that this factor is necessary to cancel a lowest-order $Z$-propagator.

In contrast to the photon case the light fermion contributions to the $Z$ self-energy depend on the Higgs mass. Whereas the contributions from the three tadpole graphs are compensated by the usual choice of the renormalization constant, the first two graphs in fig. 8.3 give a $p^{2}$-dependent contribution to the $Z$ self-energy. We obtain for the first graph

$$
\begin{aligned}
\Sigma_{T, H}^{Z Z,(2)}\left(p^{2}\right)= & \frac{e^{4}(2-D)\left(9-12 s_{W}^{2}+8 s_{W}^{4}\right)}{(4 \pi)^{4}(1-D)^{2} 48 p^{2} c_{W}^{4} s_{W}^{4}}\left\{\left[(3-D)\left(p^{2}-m_{H}^{2}\right)+(2-D) m_{Z}^{2}\right] T_{13^{\prime} 4^{\prime}}\left(m_{Z}^{2}\right)\right. \\
& +m_{Z}^{2} T_{23^{\prime} 4^{\prime}}\left(m_{H}^{2}\right)-2 m_{Z}^{2}\left[m_{H}^{2}-m_{Z}^{2}+(3-2 D) p^{2}\right] T_{123^{\prime} 4^{\prime}}\left(m_{Z}^{2}, m_{H}^{2}\right) \\
& \left.+m_{Z}^{2}\left[\left(m_{H}^{2}-m_{Z}^{2}\right)^{2}-2 p^{2}\left(m_{H}^{2}+(3-2 D) m_{Z}^{2}\right)+p^{4}\right] T_{1123^{\prime} 4^{\prime}}\left(m_{Z}^{2}, m_{Z}^{2}, m_{H}^{2}\right)\right\},
\end{aligned}
$$

which is obviously gauge invariant. The subscript $H$ indicates the dependence on the Higgs mass which appears in the scalar integrals and as coefficients $m_{H}^{2}$ and $m_{H}^{4}$, respectively. 
(a)

(b)

Figure 8.4: The light fermion contributions to the $W$ self-energy 
The result for the second graph in fig. 8.3 is derived from (8.12) by using the appropriate coupling of the $u$-quark.

The result for the three tadpole graphs reads

$$
\Sigma_{T, \text { tad }}^{Z Z,(2)}=\frac{e^{4}(2-D)^{2} m_{Z}^{2}}{(4 \pi)^{4} 12 m_{H}^{2} s_{W}^{4}}\left\{18 T_{13^{\prime} 4^{\prime}}\left(m_{W}^{2}\right)+\left[11 \frac{\left(m_{W}^{2}-m_{Z}^{2}\right)^{2}}{m_{W}^{4}}+9\right] T_{13^{\prime} 4^{\prime}}\left(m_{Z}^{2}\right)\right\}
$$

showing that they are gauge invariant. This is also true for each of the graphs separately.

In the study of the $\gamma Z$-mixing self-energy $\Sigma^{\gamma Z,(2)}\left(p^{2}\right)$ no new features appear. The diagrams are of the same type as for the photon, i.e. we have contributions from the 27 graphs shown in fig. 8.2 with one of the external photons substituted by a $Z$-boson. Since the results have precisely the same structure as in the case of the photon we do not list them in detail.

The graphs relevant for the $W^{ \pm}$self-energy are shown in fig. 8.4. As can be seen in fig. 8.4a there is no natural way to make a subdivision into "neutral" and "charged current" graphs. Like in the case of the $Z$ self-energy we get contributions from diagrams depending on the Higgs mass. These are shown in fig. 8.4 b.

We first consider the 41 graphs listed in fig. 8.4a. The result in 't Hooft-Feynman gauge, i.e. $\xi_{\gamma}=\xi_{Z}=\xi_{W}=1$, is given in the appendix. Inspection of the integrals appearing in (A.1) shows that the only integral type not present in the results of the neutral gauge bosons is $T_{123^{\prime} 4^{\prime} 5^{\prime}}\left(m_{Z}^{2}, m_{W}^{2}\right)$. Like the integrals considered above it can be solved analytically. The result is given in [13].

The result for general values of the gauge parameters is very lengthy and will not be presented here. It depends on all three gauge parameters $\xi_{\gamma}, \xi_{Z}$ and $\xi_{W}$. Therefore in this case also the neutral gauge bosons yield a gauge dependent contribution. In analogy to the $Z$ self-energy all gauge dependent terms involving the basic two-loop integrals are proportional to $\left(p^{2}-m_{W}^{2}\right)$. The integrals appearing in this result are the same as in the case of the 't Hooft-Feynman gauge (see (A.1)).

The results for the Higgs-dependent graphs in fig. 8.4b are of the same form as those obtained for the $Z$ self-energy in (8.12) and (8.13), where in (8.12) $m_{Z}$ has to be substituted by $m_{W}$.

\section{Conclusion}

Radiative corrections are necessary for the comparison between theory and precision measurements. Whereas the methods for performing one-loop calculations in massive gauge theories are well established, so far no complete treatment of irreducible two-loop corrections has been achieved. In the SM these corrections are generally expected to be small, but a detailed analysis is necessary to determine their actual value.

In this paper we presented a technique for reducing two-loop self-energies to standard scalar integrals in general massive gauge theories. It makes use of the tensor structure of the two-loop integrals, the symmetries of the scalar integrals and certain integral relations. The results are valid for all values of the invariant momentum $p^{2}$, the particle masses, the space-time dimension $D$ and the gauge parameters. Features like gauge invariance or 
transversality are displayed directly at the algebraic level. We explicitly verified SlavnovTaylor identities valid for the neutral gauge boson system of the SM by calculating several thousand Feynman diagrams.

As an application we treated the light fermion contributions to the gauge boson selfenergies in the SM. For the self-energies of the neutral gauge bosons the contributions associated with the exchange of $\gamma$ and $Z$ in the fermion loop are gauge invariant. The $W$-exchange yields gauge dependent contributions. At the pole, i.e. at $p^{2}=m_{Z}^{2}$ for the $Z$ self-energy, the gauge dependence of the generic two-loop contributions vanishes. The photon self-energy gives zero for $p^{2}=0$ as required by the Ward identity. The $Z$ selfenergy acquires a Higgs mass dependent contribution even in the light fermion case. We showed that it is gauge invariant.

The $W$ self-energy receives gauge dependent contributions from all intermediate vector bosons. As in the case of the $Z$ self-energy the generic two-loop contributions are gauge invariant at the pole, i.e. for $p^{2}=m_{W}^{2}$. The Higgs-dependent graphs form a gauge invariant subset.

All standard scalar integrals needed for the treatment of the light fermion contributions to the gauge boson self-energies can be solved analytically. We performed these calculations and obtained expressions in terms of polylogarithmic functions. The results will be presented in a related paper [13].

We would like to thank F.A. Berends, W.L. van Neerven and J.B. Tausk for fruitful discussions, R. Mertig for his contributions to the program TwoCalc and for providing FeynCalc and J. Küblbeck and H. Eck for help concerning FeynArts and its use to create the pictures included in this paper.

\section{A Result for the $W$ self-energy}

The contribution from the 41 graphs listed in fig. 8.4 a to the two-loop self-energy of the $W$-boson is given by

$$
\begin{aligned}
& \left.\Sigma_{T}^{W W,(2)}\left(p^{2}\right)\right|_{\xi_{\gamma}=\xi_{Z}=\xi_{W}=1}=\frac{e^{4}(2-D)}{(4 \pi)^{4}(1-D)^{2} 12 m_{W}^{2} m_{Z}^{2} s_{W}^{4}}\left\{18\left(\frac{1}{D}-1\right)\left(4-6 D+D^{2}\right) m_{W}^{2} m_{Z}^{2}\right. \\
& \quad \times A_{0}\left(m_{W}^{2}\right) B_{0}\left(p^{2} ; 0,0\right)+\left(\frac{1}{D}-1\right)\left(4-6 D+D^{2}\right)\left[\left(m_{W}^{2}-m_{Z}^{2}\right)^{2}+9 m_{W}^{4}\right] A_{0}\left(m_{Z}^{2}\right) B_{0}\left(p^{2} ; 0,0\right) \\
& \quad+\frac{1}{2}(1-D) m_{Z}^{2}\left[16 m_{W}^{4}+4 m_{W}^{2} m_{Z}^{2}-2 m_{Z}^{4}+10(7-D) m_{W}^{2} p^{2}-(7-D) m_{Z}^{2} p^{2}\right] B_{0}^{2}\left(p^{2} ; 0,0\right) \\
& \quad+36(1-D) m_{W}^{2}\left(m_{W}^{2}-m_{Z}^{2}\right)\left(m_{W}^{2}+p^{2}\right) B_{0}\left(p^{2} ; 0,0\right) B_{0}\left(p^{2} ; 0, m_{W}^{2}\right)-36(1-D) m_{W}^{4}\left(m_{W}^{2}\right. \\
& \left.\quad+m_{Z}^{2}+p^{2}\right) B_{0}\left(p^{2} ; 0,0\right) B_{0}\left(p^{2} ; m_{W}^{2}, m_{Z}^{2}\right)+\frac{9}{2} \frac{m_{Z}^{2}}{p^{2}}\left[(4-D) m_{W}^{4}-(3-D) m_{W}^{2} m_{Z}^{2}+\left(\frac{1}{D}-1\right)\right. \\
& \left.\quad \times\left(16-37 D+20 D^{2}-4 D^{3}\right) m_{W}^{2} p^{2}-2(3-2 D)(3-D) p^{4}\right] T_{13^{\prime} 4^{\prime}}\left(m_{W}^{2}\right)-\frac{1}{2 m_{Z}^{4} p^{2}}[40(5 \\
& \quad-D) m_{W}^{10}-4\left(264-141 D+20 D^{2}\right) m_{W}^{8} m_{Z}^{2}+6\left(279-181 D+28 D^{2}\right) m_{W}^{6} m_{Z}^{4}-6(191 \\
& \left.\quad-134 D+22 D^{2}\right) m_{W}^{4} m_{Z}^{6}+11\left(31-23 D+4 D^{2}\right) m_{W}^{2} m_{Z}^{8}-11(2-D) m_{Z}^{10}-80(2-D)(5
\end{aligned}
$$


$-D) m_{W}^{8} p^{2}+8\left(308-291 D+91 D^{2}-10 D^{3}\right) m_{W}^{6} m_{Z}^{2} p^{2}-4\left(\frac{20}{D}+543-570 D+200 D^{2}\right.$ $\left.-22 D^{3}\right) m_{W}^{4} m_{Z}^{4} p^{2}+2\left(\frac{8}{D}+409-448 D+174 D^{2}-22 D^{3}\right) m_{W}^{2} m_{Z}^{6} p^{2}-\left(\frac{1}{D}+1\right)(8+5 D$ $\left.-2 D^{2}\right) m_{Z}^{8} p^{2}+40(3-2 D)(5-D) m_{W}^{6} p^{4}-4(64-11 D)(3-2 D) m_{W}^{4} m_{Z}^{2} p^{4}+22(3-2 D)$ $\left.(5-D) m_{W}^{2} m_{Z}^{4} p^{4}\right] T_{13^{\prime} 4^{\prime}}\left(m_{Z}^{2}\right)+\frac{m_{W}^{2}}{p^{2}}\left(m_{W}^{2}-m_{Z}^{2}\right)\left[9 m_{W}^{2}+\left(33-37 D+13 D^{2}\right) p^{2}\right] T_{2^{\prime} 3^{\prime} 4^{\prime}}$ $-5(1-D)(10-D) m_{W}^{2}\left(m_{W}^{2}-m_{Z}^{2}\right) p^{2} T_{1^{\prime} 2^{\prime} 3^{\prime} 4^{\prime}}+18 \frac{m_{W}^{2}}{p^{2}}\left(m_{W}^{2}-m_{Z}^{2}\right)\left[(2-D)\left(m_{W}^{4}-p^{4}\right)\right.$ $\left.-4(1-D) m_{W}^{2} p^{2}\right] T_{12^{\prime} 3^{\prime} 4^{\prime}}\left(m_{W}^{2}\right)+9 \frac{m_{W}^{2}}{p^{2}}\left(m_{W}^{2}-m_{Z}^{2}\right)\left(m_{W}^{2}+p^{2}\right)\left[(3-2 D)\left(m_{W}^{4}+p^{4}\right)-2(5\right.$ $\left.-4 D) m_{W}^{2} p^{2}\right] T_{112^{\prime} 3^{\prime} 4^{\prime}}\left(m_{W}^{2}, m_{W}^{2}\right)+\frac{m_{Z}^{2}}{2 p^{2}}\left[2 m_{W}^{4}-22 m_{W}^{2} m_{Z}^{2}+11 m_{Z}^{4}+18\left(\frac{16}{D}-31+18 D\right.\right.$ $\left.\left.-4 D^{2}\right) m_{W}^{2} p^{2}\right] T_{23^{\prime} 4^{\prime}}\left(m_{W}^{2}\right)+\frac{2 m_{W}^{2}}{m_{Z}^{4} p^{2}}\left(m_{W}^{2}-m_{Z}^{2}\right)\left[20 m_{W}^{8}-(71-20 D) m_{W}^{6} m_{Z}^{2}+(51-20 D)\right.$ $m_{W}^{4} m_{Z}^{4}-20(5-2 D) m_{W}^{6} p^{2}+(135-62 D) m_{W}^{4} m_{Z}^{2} p^{2}-22(2-D) m_{W}^{2} m_{Z}^{4} p^{2}+20(7$ $-4 D) m_{W}^{4} p^{4}-(17+16 D) m_{W}^{2} m_{Z}^{2} p^{4}-3(17-14 D) m_{Z}^{4} p^{4}-20(3-2 D) m_{W}^{2} p^{6}+11(3$ $\left.-2 D) m_{Z}^{2} p^{6}\right] T_{1^{\prime} 23^{\prime} 4^{\prime}}\left(m_{W}^{2}\right)+\frac{20 m_{W}^{2}}{m_{Z}^{2} p^{2}}\left(m_{W}^{2}-m_{Z}^{2}\right)^{2}\left(m_{W}^{2}-p^{2}\right)\left(m_{W}^{2}-(3-2 D) p^{2}\right)\left[T_{1^{\prime} 1^{\prime} 3^{\prime} 4^{\prime}}\right.$ $\left.+\left(m_{W}^{2}-p^{2}\right) T_{1^{\prime} 1^{\prime} 23^{\prime} 4^{\prime}}\left(m_{W}^{2}\right)\right]-\frac{1}{m_{Z}^{4} p^{2}}\left[40 m_{W}^{12}-2(91-20 D) m_{W}^{10} m_{Z}^{2}+4(61-20 D) m_{W}^{8} m_{Z}^{4}\right.$ $-2(28-9 D) m_{W}^{6} m_{Z}^{6}-4(28-11 D) m_{W}^{4} m_{Z}^{8}+11(7-2 D) m_{W}^{2} m_{Z}^{10}-11 m_{Z}^{12}-40(5-2 D)$ $\times m_{W}^{10} p^{2}+2(235-102 D) m_{W}^{8} m_{Z}^{2} p^{2}-2(179-84 D) m_{W}^{6} m_{Z}^{4} p^{2}+2(11+12 D) m_{W}^{4} m_{Z}^{6} p^{2}$ $-22(3-2 D) m_{W}^{2} m_{Z}^{8} p^{2}+11(3-2 D) m_{Z}^{10} p^{2}+40(7-4 D) m_{W}^{8} p^{4}-2(157-64 D) m_{W}^{6} m_{Z}^{2} p^{4}$ $-4(17-29 D) m_{W}^{4} m_{Z}^{4} p^{4}+66(1-D) m_{W}^{2} m_{Z}^{6} p^{4}-40(3-2 D) m_{W}^{6} p^{6}+62(3-2 D)$ $\left.\times m_{W}^{4} m_{Z}^{2} p^{6}-22(3-2 D) m_{W}^{2} m_{Z}^{4} p^{6}\right] T_{123^{\prime} 4^{\prime}}\left(m_{Z}^{2}, m_{W}^{2}\right)+\frac{1}{2 m_{Z}^{2} p^{2}}\left(11\left(m_{W}^{2}-m_{Z}^{2}\right)^{2}+9 m_{W}^{4}\right)$ $\times\left[2 m_{W}^{8}-4(3-D) m_{W}^{6} m_{Z}^{2}+(19-8 D) m_{W}^{4} m_{Z}^{4}-2(5-2 D) m_{W}^{2} m_{Z}^{6}+m_{Z}^{8}-2(5-2 D)\right.$ $m_{W}^{6} p^{2}+8(2-D) m_{W}^{4} m_{Z}^{2} p^{2}+4(6-5 D) m_{W}^{2} m_{Z}^{4} p^{2}-2(3-2 D) m_{Z}^{6} p^{2}+2(7-4 D) m_{W}^{4} p^{4}$ $\left.+12(1-D) m_{W}^{2} m_{Z}^{2} p^{4}+m_{Z}^{4} p^{4}-2(3-2 D) m_{W}^{2} p^{6}\right] T_{1123^{\prime} 4^{\prime}}\left(m_{Z}^{2}, m_{Z}^{2}, m_{W}^{2}\right)-\frac{1}{2 p^{2}}\left[18 m_{W}^{6}\right.$ $-9 m_{W}^{4} m_{Z}^{2}-2\left(\frac{80}{D}-183+122 D-28 D^{2}\right) m_{W}^{4} p^{2}+2\left(\frac{1}{D}-1\right)\left(8-4 D+D^{2}\right)\left(2 m_{W}^{2}\right.$ $\left.\left.-m_{Z}^{2}\right) m_{Z}^{2} p^{2}\right] T_{23^{\prime} 4^{\prime}}\left(m_{Z}^{2}\right)-9 \frac{m_{W}^{4}}{p^{2}}\left[2(2-D) m_{W}^{4}-(7-4 D) m_{W}^{2} m_{Z}^{2}+(3-2 D) m_{Z}^{4}-8(1\right.$ $\left.-D) m_{W}^{2} p^{2}-(3-2 D) m_{Z}^{2} p^{2}-2(2-D) p^{4}\right] T_{123^{\prime} 4^{\prime}}\left(m_{W}^{2}, m_{Z}^{2}\right)-\frac{9 m_{W}^{4}}{2 p^{2}}\left[2(3-2 D) m_{W}^{6}\right.$ $-(13-8 D) m_{W}^{4} m_{Z}^{2}+4(2-D) m_{W}^{2} m_{Z}^{4}-m_{Z}^{6}-2(7-6 D) m_{W}^{4} p^{2}-6(3-2 D) m_{W}^{2} m_{Z}^{2} p^{2}$ $\left.+4(2-D) m_{Z}^{4} p^{2}-2(7-6 D) m_{W}^{2} p^{4}-(13-8 D) m_{Z}^{2} p^{4}+2(3-2 D) p^{6}\right]$ $\times T_{1123^{\prime} 4^{\prime}}\left(m_{W}^{2}, m_{W}^{2}, m_{Z}^{2}\right)-18\left(\frac{1}{D}-1\right)\left(4-8 D+D^{2}\right) m_{W}^{2} m_{Z}^{2}\left(m_{W}^{2}+p^{2}\right) T_{1^{\prime} 2^{\prime} 34^{\prime}}\left(m_{W}^{2}\right)-\left(\frac{1}{D}\right.$ 


$$
\begin{aligned}
& -1)\left(4-8 D+D^{2}\right)\left(m_{Z}^{2}+p^{2}\right)\left(\left(m_{W}^{2}-m_{Z}^{2}\right)^{2}+9 m_{W}^{4}\right) T_{1^{\prime} 2^{\prime} 34^{\prime}}\left(m_{Z}^{2}\right)-8(1-D) m_{W}^{2}\left(m_{W}^{2}\right. \\
& \left.-m_{Z}^{2}\right) p^{4} T_{1^{\prime} 2^{\prime} 3^{\prime} 4^{\prime} 5^{\prime}}^{2}-72(1-D) m_{W}^{4}\left(m_{W}^{2}-m_{Z}^{2}\right) p^{2} T_{12^{\prime} 3^{\prime} 4^{\prime} 5^{\prime}}\left(m_{W}^{2}\right)+72(1-D) m_{W}^{4}\left(m_{W}^{2} m_{Z}^{2}\right. \\
& \left.+m_{W}^{2} p^{2}+m_{Z}^{2} p^{2}\right) T_{123^{\prime} 4^{\prime} 5^{\prime}}\left(m_{Z}^{2}, m_{W}^{2}\right)+\frac{1}{2}(1-D)\left(4 m_{W}^{2}-m_{Z}^{2}\right)\left(2 m_{W}^{2}+m_{Z}^{2}\right)\left(2 m_{Z}^{4}+(8\right. \\
& \text {-D) } \left.\left.m_{Z}^{2} p^{2}+2 p^{4}\right) T_{1^{\prime} 2^{\prime} 34^{\prime} 5^{\prime}}\left(m_{Z}^{2}\right)\right\}
\end{aligned}
$$

where the 't Hooft-Feynman gauge was used.

\section{B Integral relations}

We list here some relations needed for the calculations performed in this paper. The reduction formula for the integral $Y_{2345}^{11}$ reads

$$
\begin{aligned}
Y_{2345}^{11}= & 2\left(m_{2}^{2}+p^{2}\right) Y_{2345}^{1}+A_{0}\left(m_{3}^{2}\right) A_{0}\left(m_{5}^{2}\right)-\left(m_{2}^{2}-m_{3}^{2}\right) A_{0}\left(m_{3}^{2}\right) B_{0}\left(p^{2} ; m_{4}^{2}, m_{5}^{2}\right) \\
& +\left(m_{4}^{2}-p^{2}\right)\left[T_{355^{\prime}}+\left(m_{4}^{2}-p^{2}\right) T_{3455^{\prime}}\right]-\left(m_{2}^{2}+p^{2}\right)^{2} T_{2345}+m_{2}^{2}\left[T_{234}-T_{235}-\left(2 m_{4}^{2}\right.\right. \\
& \left.\left.-m_{5}^{2}-2 p^{2}\right) T_{2345}\right]+m_{2}^{2}\left(m_{4}^{2}-p^{2}\right)\left[T_{2355^{\prime}}+\left(m_{4}^{2}-p^{2}\right) T_{23455^{\prime}}\right] \\
& +\frac{p^{2}}{1-D}\left\{T_{234}-\left[A_{0}\left(m_{2}^{2}\right)+A_{0}\left(m_{3}^{2}\right)\right] B_{0}\left(p^{2} ; m_{4}^{2}, m_{5}^{2}\right)-\left(m_{2}^{2}-m_{3}^{2}\right)\left[T_{2455^{\prime}}-T_{3455^{\prime}}\right]\right. \\
& \left.-\left(2 m_{2}^{2}+2 m_{3}^{2}-m_{5}^{2}\right) T_{2345}+\left(m_{2}^{2}-m_{3}^{2}\right)^{2} T_{23455^{\prime}}\right\} \\
& -\frac{D}{4(1-D)}\left\{Y_{234}^{5}-A_{0}\left(m_{2}^{2}\right) A_{0}\left(m_{3}^{2}\right)-\left[A_{0}\left(m_{2}^{2}\right)+A_{0}\left(m_{3}^{2}\right)\right]\left[A_{0}\left(m_{4}^{2}\right)-A_{0}\left(m_{5}^{2}\right)\right]\right. \\
& -\left(2 m_{2}^{2}+2 m_{3}^{2}+2 m_{4}^{2}-m_{5}^{2}-2 p^{2}\right) T_{234}+\left(2 m_{2}^{2}+2 m_{3}^{2}+m_{4}^{2}-m_{5}^{2}-p^{2}\right) T_{235} \\
& -\left(m_{2}^{2}-m_{3}^{2}-2 m_{4}^{2}+m_{5}^{2}+2 p^{2}\right) T_{245}+\left(m_{2}^{2}-m_{3}^{2}+2 m_{4}^{2}-m_{5}^{2}-2 p^{2}\right) T_{345} \\
& +\left(m_{2}^{2}-m_{3}^{2}-m_{4}^{2}+p^{2}\right) T_{255^{\prime}}-\left(m_{2}^{2}-m_{3}^{2}+m_{4}^{2}-p^{2}\right) T_{355^{\prime}} \\
& +\left[\left(m_{4}^{2}-m_{5}^{2}-p^{2}\right)^{2}+2\left(m_{2}^{2}+m_{3}^{2}\right)\left(2 m_{4}^{2}-m_{5}^{2}-2 p^{2}\right)+\left(m_{2}^{2}-m_{3}^{2}\right)^{2}\right] T_{2345} \\
& -\left[2\left(m_{2}^{2}+m_{3}^{2}\right)\left(m_{4}^{2}-p^{2}\right)+\left(m_{2}^{2}-m_{3}^{2}\right)^{2}\right] T_{2355^{\prime}}-\left[\left(m_{4}^{2}-p^{2}\right)^{2}-2\left(m_{2}^{2}-m_{3}^{2}\right)\left(m_{4}^{2}\right.\right. \\
& \left.\left.-p^{2}\right)\right] T_{2455^{\prime}}-\left[\left(m_{4}^{2}-p^{2}\right)^{2}+2\left(m_{2}^{2}-m_{3}^{2}\right)\left(m_{4}^{2}-p^{2}\right)\right] T_{3455^{\prime}}-\left(m_{2}^{2}-m_{3}^{2}\right)\left(m_{4}^{2}\right. \\
& \left.-p^{2}\right)\left[T_{255^{\prime} 5^{\prime}}-T_{355^{\prime} 5^{\prime}}\right]-\left[2\left(m_{2}^{2}-m_{3}^{2}\right)^{2}\left(m_{4}^{2}-p^{2}\right)+2\left(m_{2}^{2}+m_{3}^{2}\right)\left(m_{4}^{2}-p^{2}\right)^{2}\right] T_{23455^{\prime}} \\
& +\left(m_{2}^{2}-m_{3}^{2}\right)^{2}\left(m_{4}^{2}-p^{2}\right) T_{2355^{\prime} 5^{\prime}}-\left(m_{2}^{2}-m_{3}^{2}\right)\left(m_{4}^{2}-p^{2}\right)^{2}\left[T_{2455^{\prime} 5^{\prime}}-T_{3455^{\prime} 5^{\prime}}\right] \\
& \left.+\left(m_{2}^{2}-m_{3}^{2}\right)^{2}\left(m_{4}^{2}-p^{2}\right)^{2} T_{23455^{\prime} 5^{\prime}}\right\} . \\
&
\end{aligned}
$$

From this relation the formula for the integral $Y_{23455}^{11}$ can be obtained by taking the derivative with respect to $m_{5}^{2}$.

For the integral $Y_{234}^{11}$ we get

$Y_{234}^{11}=\frac{1}{3(4-5 D)(4-3 D)}\left\{\left[(-4+5 D) D m_{2}^{2}-(16-11 D) D m_{3}^{2}+(5-4 D)(4-3 D)\right.\right.$ 


$$
\begin{aligned}
& \left.\times D m_{4}^{2}+\left(48-100 D+65 D^{2}-12 D^{3}\right) p^{2}\right] A_{0}\left(m_{2}^{2}\right) A_{0}\left(m_{3}^{2}\right)+\left[\left(48-76 D+29 D^{2}\right) m_{2}^{2}\right. \\
& \left.-(4-5 D) D m_{3}^{2}-(16-11 D) D m_{4}^{2}-(4-5 D) D p^{2}\right] A_{0}\left(m_{2}^{2}\right) A_{0}\left(m_{4}^{2}\right)+[(-4+3 D) \\
& \left.\times D(1+D) m_{2}^{2}+\left(48-76 D+29 D^{2}\right) m_{3}^{2}-(4-5 D) D m_{4}^{2}-D\left(16-17 D+3 D^{2}\right) p^{2}\right] \\
& \times A_{0}\left(m_{3}^{2}\right) A_{0}\left(m_{4}^{2}\right)+\left[\left(16-36 D+17 D^{2}\right) m_{2}^{4}+2(4-D) D m_{2}^{2} m_{3}^{2}-6 D^{2} m_{2}^{2} m_{4}^{2}+2(8\right. \\
& \left.-5 D) D m_{2}^{2} p^{2}+2 D(2+D) m_{3}^{2} p^{2}-6(2-D) D m_{4}^{2} p^{2}-\left(16-20 D+7 D^{2}\right) p^{4}\right] \\
& \times A_{0}\left(m_{3}^{2}\right) B_{0}\left(p^{2} ; 0, m_{2}^{2}\right)+\left[\left(-16+24 D-11 D^{2}\right) m_{2}^{4}-2(4-D) D m_{2}^{2} m_{3}^{2}+6 D^{2} m_{2}^{2} m_{4}^{2}\right. \\
& \left.+2(4-D) D m_{2}^{2} p^{2}-2 D(2+D) m_{3}^{2} p^{2}+6(2-D) D m_{4}^{2} p^{2}+\left(16-32 D+13 D^{2}\right) p^{4}\right] \\
& \times A_{0}\left(m_{4}^{2}\right) B_{0}\left(p^{2} ; 0, m_{2}^{2}\right)-2\left(m_{3}^{2}-p^{2}\right)\left[\left(8-20 D+11 D^{2}\right) m_{2}^{2}-(4-3 D) D m_{3}^{2}-(2\right. \\
& \left.-D) D m_{4}^{2}+(2-3 D)(4-3 D) p^{2}\right]\left[A_{0}\left(m_{2}^{2}\right)-A_{0}\left(m_{4}^{2}\right)\right] B_{0}\left(p^{2} ; 0, m_{3}^{2}\right)-2[(16-20 D \\
& \left.+3 D^{2}\right) m_{2}^{2} m_{4}^{2}-(12-11 D) D m_{3}^{2} m_{4}^{2}-(8-7 D) D m_{4}^{4}+\left(8-4 D-3 D^{2}\right) m_{2}^{2} p^{2}-(24 \\
& \left.\left.-36 D+11 D^{2}\right) m_{3}^{2} p^{2}+4(1-D)(4-D) m_{4}^{2} p^{2}-\left(16-28 D+11 D^{2}\right) p^{4}\right] A_{0}\left(m_{2}^{2}\right) \\
& \times B_{0}\left(p^{2} ; 0, m_{4}^{2}\right)+2\left[\left(16-20 D+3 D^{2}\right) m_{2}^{2} m_{4}^{2}-(12-11 D) D m_{3}^{2} m_{4}^{2}+(24-44 D\right. \\
& \left.+19 D^{2}\right) m_{4}^{4}+\left(8-4 D-3 D^{2}\right) m_{2}^{2} p^{2}-\left(24-36 D+11 D^{2}\right) m_{3}^{2} p^{2}-4(8-5 D)(1-D) \\
& \left.m_{4}^{2} p^{2}+\left(8-8 D+D^{2}\right) p^{4}\right] A_{0}\left(m_{3}^{2}\right) B_{0}\left(p^{2} ; 0, m_{4}^{2}\right)-3 D^{2}\left(m_{3}^{2}-m_{4}^{2}\right)\left(m_{2}^{2}-p^{2}\right)^{2}\left[A_{0}\left(m_{3}^{2}\right)\right. \\
& \left.-A_{0}\left(m_{4}^{2}\right)\right] B_{0}^{\prime}\left(p^{2} ; 0, m_{2}^{2}\right)+12(1-D) D\left(m_{2}^{2}-m_{3}^{2}\right)\left(m_{4}^{2}-p^{2}\right)^{2}\left[A_{0}\left(m_{2}^{2}\right)-A_{0}\left(m_{3}^{2}\right)\right] \\
& \times B_{0}^{\prime}\left(p^{2} ; 0, m_{4}^{2}\right)+2\left[(8-9 D)(2-D) m_{2}^{4}-4(1-D)(4-D) m_{2}^{2} m_{3}^{2}+(6-5 D) D m_{3}^{4}\right. \\
& +\left(24-56 D+37 D^{2}-6 D^{3}\right) m_{2}^{2} m_{4}^{2}-(4-3 D)(1-2 D) D m_{3}^{2} m_{4}^{2}-\left(8-20 D+19 D^{2}\right. \\
& \left.\left.-6 D^{3}\right) m_{2}^{2} p^{2}-(4-3 D)\left(4-7 D+2 D^{2}\right) m_{3}^{2} p^{2}\right] T_{134^{\prime}}\left(m_{3}^{2}, m_{2}^{2}\right)+2\left(m_{2}^{2}-m_{4}^{2}\right) \\
& \times\left[\left(8-20 D+11 D^{2}\right) m_{2}^{2}-(4-3 D) D m_{3}^{2}-(2-D) D m_{4}^{2}+(2-3 D)(4-3 D) p^{2}\right] \\
& \times T_{134^{\prime}}\left(m_{4}^{2}, m_{2}^{2}\right)+\left[(-4+3 D)(1-D)(4+D) m_{2}^{2} m_{3}^{2}-(8-5 D) D m_{3}^{4}+(1-D)(16\right. \\
& \left.-20 D+3 D^{2}\right) m_{2}^{2} m_{4}^{2}+2(4-D) D m_{3}^{2} m_{4}^{2}-3 D^{2} m_{4}^{4}-(4-3 D)(1-D)(4-D) m_{3}^{2} p^{2} \\
& \left.+(1-D)\left(16-4 D-3 D^{2}\right) m_{4}^{2} p^{2}\right] T_{134^{\prime}}\left(m_{4}^{2}, m_{3}^{2}\right)+\left[(-4+5 D) D m_{2}^{4}+8(3-2 D)(2\right. \\
& -D) m_{2}^{2} m_{3}^{2}-(4-5 D) D m_{3}^{4}-4(4-5 D)(3-D) m_{2}^{2} m_{4}^{2}-2(22-17 D) D m_{3}^{2} m_{4}^{2} \\
& -(4-5 D) D m_{4}^{4}-2(22-17 D) D m_{2}^{2} p^{2}-4(4-5 D)(3-D) m_{3}^{2} p^{2}+8(3-2 D)(2 \\
& \left.-D) m_{4}^{2} p^{2}-(4-5 D) D p^{4}\right] T_{234}\left(m_{4}^{2}, m_{3}^{2}, m_{2}^{2}\right)+2\left[\left(16-20 D+3 D^{2}\right) m_{2}^{4} m_{4}^{2}-8(1\right. \\
& -D)(2+D) m_{2}^{2} m_{3}^{2} m_{4}^{2}+(12-11 D) D m_{3}^{4} m_{4}^{2}+(12-13 D)(2-D) m_{2}^{2} m_{4}^{4}+(14 \\
& -13 D) D m_{3}^{2} m_{4}^{4}+\left(8-4 D-3 D^{2}\right) m_{2}^{4} p^{2}-8(1-D)(4-D) m_{2}^{2} m_{3}^{2} p^{2}+(24-36 D \\
& \left.+11 D^{2}\right) m_{3}^{4} p^{2}-8(1-D)(4-D) m_{2}^{2} m_{4}^{2} p^{2}-8(1-D)(2+D) m_{3}^{2} m_{4}^{2} p^{2}+(8-2 D \\
& \left.\left.-5 D^{2}\right) m_{2}^{2} p^{4}+\left(16-22 D+5 D^{2}\right) m_{3}^{2} p^{4}\right] T_{1^{\prime} 234}\left(m_{4}^{2}, m_{3}^{2}, m_{2}^{2}\right)-12(1-D) D\left(m_{2}^{2}\right. \\
& \left.-m_{3}^{2}\right)^{2}\left(m_{4}^{2}-p^{2}\right)^{2} T_{1^{\prime} 1^{\prime} 234}\left(m_{4}^{2}, m_{3}^{2}, m_{2}^{2}\right)+2\left(m_{2}^{2}-m_{4}^{2}\right)\left(m_{3}^{2}-p^{2}\right)\left[\left(8-20 D+11 D^{2}\right)\right. \\
& \left.\times m_{2}^{2}-(4-3 D) D m_{3}^{2}-(2-D) D m_{4}^{2}+(2-3 D)(4-3 D) p^{2}\right] T_{1^{\prime} 234}\left(m_{3}^{2}, m_{4}^{2}, m_{2}^{2}\right) \\
& -2\left[\left(8-12 D+7 D^{2}\right) m_{2}^{4} m_{3}^{2}+(4-D) D m_{2}^{2} m_{3}^{4}-(4-7 D)(2-D) m_{2}^{4} m_{4}^{2}-2 D(2\right.
\end{aligned}
$$




$$
\begin{aligned}
& +D) m_{2}^{2} m_{3}^{2} m_{4}^{2}+3 D^{2} m_{2}^{2} m_{4}^{4}-2 D(2+D) m_{2}^{2} m_{3}^{2} p^{2}+D(2+D) m_{3}^{4} p^{2}-2(4-D) \\
& \times D m_{2}^{2} m_{4}^{2} p^{2}-2(4-D) D m_{3}^{2} m_{4}^{2} p^{2}+3(2-D) D m_{4}^{4} p^{2}-\left(8-16 D+5 D^{2}\right) m_{3}^{2} p^{4} \\
& \left.+\left(8-10 D+5 D^{2}\right) m_{4}^{2} p^{4}\right] T_{1^{\prime} 234}\left(m_{2}^{2}, m_{4}^{2}, m_{3}^{2}\right) \\
& \left.+3 D^{2}\left(m_{3}^{2}-m_{4}^{2}\right)^{2}\left(m_{2}^{2}-p^{2}\right)^{2} T_{1^{\prime} 1^{\prime} 234}\left(m_{2}^{2}, m_{4}^{2}, m_{3}^{2}\right)\right\}
\end{aligned}
$$

The integral $Y_{234}^{15}$ is expressible through $Y_{234}^{11}$ and integrals of simpler structure via

$$
\begin{aligned}
Y_{234}^{15}= & -\frac{1}{2} Y_{234}^{11}+\frac{1}{2}\left\{Y_{23}^{1}+Y_{24}^{1}+Y_{34}^{1}+\left(m_{2}^{2}+m_{3}^{2}+m_{4}^{2}+p^{2}\right) Y_{234}^{1}+\left(m_{2}^{2}-p^{2}\right) A_{0}\left(m_{2}^{2}\right)\right. \\
& \left.\times\left[A_{0}\left(m_{3}^{2}\right)-A_{0}\left(m_{4}^{2}\right)\right]-\left(m_{3}^{2}-m_{4}^{2}\right) A_{0}\left(m_{3}^{2}\right) A_{0}\left(m_{4}^{2}\right)-\left(m_{2}^{2}-p^{2}\right)\left(m_{3}^{2}-m_{4}^{2}\right) T_{234}\right\} .
\end{aligned}
$$

The expression for the integral $T_{2345^{\prime} 5^{\prime}}\left(m^{2}, M^{2}, M^{2}\right)$ in terms of integrals with fewer propagators reads

$$
\begin{aligned}
& T_{2345^{\prime} 5^{\prime}}\left(m^{2}, M^{2}, M^{2}\right)=\frac{1}{D\left(m^{2}-M^{2}\right)^{2}\left(M^{2}-p^{2}\right)^{2}}\left\{( 2 - D ) \left[2 m^{2}-(4-D) M^{2}+(2\right.\right. \\
& \left.\quad-D) p^{2}\right] A_{0}\left(m^{2}\right) A_{0}\left(M^{2}\right)+2(2-D)\left(M^{2}-p^{2}\right) A_{0}^{2}\left(M^{2}\right)-2(2-D)\left(m^{2}-p^{2}\right)^{2} A_{0}\left(M^{2}\right) \\
& \quad \times B_{0}\left(p^{2} ; 0, m^{2}\right)-\left[D m^{2} M^{2}-(8-3 D) M^{4}+(4-D) m^{2} p^{2}+(4-3 D) M^{2} p^{2}\right] A_{0}\left(m^{2}\right) \\
& \quad \times B_{0}\left(p^{2} ; 0, M^{2}\right)+\left[D m^{2} M^{2}-(4-D)\left(M^{4}+\left(m^{2}-M^{2}\right) p^{2}\right)+2(2-D) p^{4}\right] A_{0}\left(M^{2}\right) \\
& \quad \times B_{0}\left(p^{2} ; 0, M^{2}\right)+D\left(m^{2}-M^{2}\right)\left(M^{2}-p^{2}\right)^{2}\left[A_{0}\left(m^{2}\right)-A_{0}\left(M^{2}\right)\right] B_{0}^{\prime}\left(p^{2} ; 0, M^{2}\right)-(2 \\
& -D)\left[(2+D) m^{2} M^{2}-(4-D) M^{4}+(2-D) m^{2} p^{2}-D M^{2} p^{2}\right] T_{235^{\prime}}\left(m^{2}, M^{2}\right) \\
& -4 M^{2}\left(m^{2}-p^{2}\right) T_{235^{\prime}}\left(M^{2}, M^{2}\right)+(8-3 D)\left(m^{2}-M^{2}\right)\left(M^{2}-p^{2}\right) T_{234}\left(m^{2}, M^{2}, M^{2}\right) \\
& \quad+\left[D\left(m^{4} M^{2}+M^{2} p^{4}\right)-(4-D)\left(m^{2}\left(M^{4}-p^{4}\right)-p^{2}\left(m^{4}-M^{4}\right)-2 M^{6}\right)\right. \\
& \left.\left.\quad-8 m^{2} M^{2} p^{2}\right] T_{2345^{\prime}}\left(m^{2}, M^{2}, M^{2}\right)-4 M^{2}\left(m^{2}-p^{2}\right)^{2} T_{1^{\prime} 234}\left(m^{2}, M^{2}, M^{2}\right)\right\} .
\end{aligned}
$$

\section{References}

[1] D.C. Kennedy, Nucl. Phys. B351 (1991) 81;

M.E. Peskin and T. Takeuchi, Phys. Rev. Lett. 65 (1990) 964;

M.E. Peskin and T. Takeuchi, SLAC-PUB-5618, (1991);

M. Golden and L. Randall, Nucl. Phys. B361 (1991) 3;

B. Holdom and J. Terning, Phys. Lett. B247 (1990) 88;

H. Georgi, Nucl. Phys. B363 (1991) 301;

G. Altarelli and R. Barbieri, Phys. Lett. B253 (1991) 161;

G. Altarelli, R. Barbieri and S. Jadach, Nucl. Phys. B369 (1992) 3

[2] D.C. Kennedy and B.W. Lynn, Nucl. Phys. B322 (1989) 1 
[3] J. van der Bij and F. Hoogeveen, Nucl. Phys. B283 (1987) 477

[4] R. Barbieri, M. Beccaria, P. Ciafaloni, G. Curci and A. Vicere, Phys. Lett. B288 (1992) 95, erratum: B312 (1993) 511; CERN-TH.6713/92 (1992)

[5] J. van der Bij and M. Veltman, Nucl. Phys. B231 (1984) 205;

J. van der Bij, Nucl. Phys. B248 (1984) 141

[6] M. Consoli, W. Hollik and F. Jegerlehner, Phys. Lett. B227 (1989) 167;

"Z Physics at LEP 1", eds.: G. Altarelli, R. Kleiss, C. Verzegnassi, CERN 89-08 (1989), Vol. 1, p. 7

[7] G. Passarino and M. Veltman, Nucl. Phys. B160 (1979) 151

[8] G. 't Hooft and M. Veltman, Nucl. Phys. B153 (1979) 365

[9] J.M. Cornwall, Phys. Rev. D26, (1982) 1453;

J. Papavassiliou, Phys. Rev. D41 (1990) 3179;

G. Degrassi and A. Sirlin, Phys. Rev. D46 (1992) 3104;

D.C. Kennedy et al., Nucl. Phys. B321, (1989) 83;

B.W. Lynn, Stanford University report SU-ITP-867 (1989);

D.C. Kennedy, FNAL Report FERMI-CONF-91/271-T (1992);

M. Kuroda, G. Moultaka und D. Schildknecht, Nucl. Phys. B350, 25 (1991)

[10] J.M. Cornwall and J. Papavassiliou, Phys. Rev. D40 (1989) 3474

[11] G. Degrassi and A. Sirlin, Nucl. Phys. B383 (1992) 73

[12] G. Weiglein, R. Mertig, R. Scharf and M. Böhm: in "New Computing Techniques in Physics Research 2, ed.: D. Perret-Gallix, World Scientific 1992, p. 617

[13] R. Scharf and J.B. Tausk, Scalar two-loop integrals for gauge boson self-energy diagrams with a massless fermion loop, (to be published)

[14] A.I. Davydychev and J.B. Tausk, Nucl. Phys. B397 (1993) 123

[15] D.J. Broadhurst, Z. Phys. C47 (1990) 115

[16] D.J. Broadhurst, Phys. Lett. B101 (1981) 423

[17] R. Scharf, Diploma Thesis, University of Würzburg, 1991

[18] D. Kreimer, Phys. Lett. B273 (1991) 277

[19] J. Küblbeck, M. Böhm and A. Denner, Comp. Phys. Comm. 60 (1990) 165;

H. Eck and J. Küblbeck, Guide to FeynArts 1.0, University of Würzburg, 1992

[20] R. Mertig, M. Böhm and A. Denner, Comp. Phys. Comm. 64 (1991) 345;

R. Mertig, Guide to FeynCalc 1.0, University of Würzburg, 1992

[21] M. Böhm, W. Hollik, and H. Spiesberger, Fortschr. Phys. 34 (1986) 687 
[22] U. Nierste, D. Müller and M. Böhm, to appear in Z. Phys. C

[23] G. Källén and A. Sabry, Dan. Mat. Fys. Medd. 29 (1955) No. 17;

R. Barbieri, E. Remiddi, Nuovo Cimento 13A (1973) 99

[24] T.H. Chang, K.J.F. Gaemers and W.L. van Neerven, Phys. Lett. B108, (1982) 222;

T.H. Chang, K.J.F. Gaemers and W.L. van Neerven, Nucl. Phys. B202 (1982) 407;

A. Djouadi and C. Verzegnassi, Phys. Lett. B195 (1987) 265;

A. Djouadi, Nuovo Cimento 100A (1988) 357;

B.A. Kniehl, Nucl. Phys. B347 (1990) 89

[25] T. Kinoshita, J. Math. Phys. 3 (1962) 650 
This figure "fig1-1.png" is available in "png" format from: http://arxiv.org/ps/hep-ph/9310358v1 
This figure "fig1-2.png" is available in "png" format from: http://arxiv.org/ps/hep-ph/9310358v1 
This figure "fig1-3.png" is available in "png" format from: http://arxiv.org/ps/hep-ph/9310358v1 
This figure "fig1-4.png" is available in "png" format from: http://arxiv.org/ps/hep-ph/9310358v1 
This figure "fig1-5.png" is available in "png" format from: http://arxiv.org/ps/hep-ph/9310358v1 
This figure "fig1-6.png" is available in "png" format from: http://arxiv.org/ps/hep-ph/9310358v1 
This figure "fig1-7.png" is available in "png" format from: http://arxiv.org/ps/hep-ph/9310358v1 
This figure "fig1-8.png" is available in "png" format from: http://arxiv.org/ps/hep-ph/9310358v1 
This figure "fig1-9.png" is available in "png" format from: http://arxiv.org/ps/hep-ph/9310358v1 
This figure "fig1-10.png" is available in "png" format from: http://arxiv.org/ps/hep-ph/9310358v1 